\title{
The Numerical Solution of Second-Order Boundary Value Problems on Nonuniform Meshes
}

\author{
By Thomas A. Manteuffel and Andrew B. White, Jr.
}

\begin{abstract}
In this paper, we examine the solution of second-order, scalar boundary value problems on nonuniform meshes. We show that certain commonly used difference schemes yield second-order accurate solutions despite the fact that their truncation error is of lower order. This result illuminates a limitation of the standard stability, consistency proof of convergence for difference schemes defined on nonuniform meshes. A technique of reducing centered-difference approximations of first-order systems to discretizations of the underlying scalar equation is developed. We treat both vertex-centered and cell-centered difference schemes and indicate how these results apply to partial differential equations on Cartesian product grids.
\end{abstract}

1. Introduction. Much attention has been paid to the numerical solution of second-order differential equations on nonuniform meshes. To begin, we consider the solution of the linear, two-point boundary value problem

$$
\begin{gathered}
y^{\prime \prime}+a(x) y^{\prime}+b(x) y=f(x), \quad x \in(0,1), \\
b_{00} y(0)+b_{01} y^{\prime}(0)=b_{02}, \\
b_{10} y(1)+b_{11} y^{\prime}(1)=b_{12},
\end{gathered}
$$

through three-point (compact-as-possible in the sense of Kreiss [15]) difference schemes on a mesh $\left\{x_{i}\right\}_{i=0}^{i=N}$. The functions $a, b$, and $f$ are assumed to be smooth. The standard mesh spacing will be written as $\Delta_{i}=x_{i}-x_{i-1}$, and we will denote a function evaluated at the mesh points by subscripts, $y_{i}$, and the vector with these entries as $Y$. The $i$ th component of $Y$ may also be written $(Y)_{i}$. We will make liberal use of the generic constant $C$ and of $\Delta=\max \left\{\Delta_{i}\right\}$.

An example of such a difference scheme is that used by Pearson [21] in his classic work on singular perturbations and by Denny and Landis [5] and de Rivas [6] in their work on mesh-selection techniques. In this method, each term in (1.1) is approximated separately on the stencil $\left(x-\Delta_{i}, x, x+\Delta_{i+1}\right)$. Approximations to the derivatives are

$$
\begin{gathered}
{\left[\frac{2}{\Delta_{i}\left(\Delta_{i}+\Delta_{i+1}\right)}, \frac{-2}{\Delta_{i} \Delta_{i+1}}, \frac{2}{\left(\Delta_{i}+\Delta_{i+1}\right) \Delta_{i+1}}\right]\left[\begin{array}{c}
y_{i-1} \\
y_{i} \\
y_{i+1}
\end{array}\right]} \\
=y^{\prime \prime}+\frac{1}{3}\left(\Delta_{i+1}-\Delta_{i}\right) y^{\prime \prime \prime}+O\left(\Delta^{2}\right)
\end{gathered}
$$

Received January 26, 1984; revised May 17, 1985.

1980 Mathematics Subject Classification. Primary 65L05, 65L10; Secondary 65L25, 40A30.

Key words and phrases. Boundary value problems, compact difference schemes, irregular grids, nonuniform mesh, difference quotients, truncation error. 


$$
\begin{gathered}
{\left[\frac{-\Delta_{i+1}}{\Delta_{i}\left(\Delta_{i}+\Delta_{i+1}\right)},\right.} \\
\left.\frac{\Delta_{i+1}-\Delta_{i}}{\Delta_{i} \Delta_{i+1}}, \frac{\Delta_{i}}{\Delta_{i+1}\left(\Delta_{i}+\Delta_{i+1}\right)}\right]\left[\begin{array}{c}
y_{i-1} \\
y_{i} \\
y_{i+1}
\end{array}\right] \\
=y^{\prime}+\frac{1}{6} \Delta_{i} \Delta_{i+1} y^{\prime \prime \prime}+O\left(\Delta^{3}\right),
\end{gathered}
$$

where each term on the right-hand side of these equations is evaluated at $x$. Throughout, the "big $O$ " notation is shorthand for terms bounded by $C \Delta_{\max }^{p}$.

This difference scheme, applied to (1.1), can be written as

$$
\begin{aligned}
\left(L_{h} V\right)_{i} \equiv & \frac{2-a_{i} \Delta_{i+1}}{\Delta_{i}\left(\Delta_{i}+\Delta_{i+1}\right)}(V)_{i-1}+\left[\frac{-2+a_{i}\left(\Delta_{i+1}-\Delta_{i}\right)}{\Delta_{i} \Delta_{i+1}}+b_{i}\right](V)_{i} \\
& +\frac{2+a_{i} \Delta_{i}}{\Delta_{i+1}\left(\Delta_{i}+\Delta_{i+1}\right)}(V)_{i+1}=f_{i}
\end{aligned}
$$

for $i=1, \ldots, N-1$. Note that this is a familiar, second-order accurate difference scheme on a uniform mesh (cf. Isaacson and Keller [11]). As usual, the truncation error is defined by replacing $V$ in (1.4) with the exact solution $Y$,

$$
\left(L_{h} Y\right)_{i}=f_{i}+(T)_{i} \text {. }
$$

In this particular case, expanding in a Taylor series about $x_{i}$ yields

$$
(T)_{i}=\frac{1}{3}\left(\Delta_{i+1}-\Delta_{i}\right) y_{i}^{\prime \prime \prime}+O\left(\Delta^{2}\right)
$$

for $i=1, \ldots, N-1$. Notice that the truncation error is not second-order unless the mesh is almost uniform.

The standard proof of convergence uses stability and consistency to imply convergence. In other words, given stability, a sufficient condition for second-order accuracy is that the truncation error be second-order. This is not a necessary condition, but it is a convenient and powerful tool. Many attempts have been made to derive schemes that are second-order accurate, i.e., yield second-order convergence in the maximum mesh size, on nonuniform meshes by forcing the truncation error to be second-order accurate. Consider, for example, the use of smoothly varying mesh functions. The notion of slowly varying meshes is natural in solving problems of this sort (cf. Chong [2], Hoffman [10]). Here the idea is to restrict the change in the mesh size so that

$$
\left(\Delta_{i+1}-\Delta_{i}\right)=O\left(\Delta^{2}\right)
$$

Clearly, then, both terms in the truncation error (1.6) will be second-order. A closely related scheme assumes that there exists a smooth mesh function, say $g(s) \in C^{2}(0,1)$, such that

$$
\begin{array}{ll}
x_{i}=g\left(s_{i}\right), & i=0, \ldots, N, \\
s_{i}=i / N, & i=0, \ldots, N .
\end{array}
$$

Such transformations have been discussed in various contexts by Ablow and Schechter [1], de Rivas [6], Davis and Flaherty [4], Hoffman [10], and White [24], among others. In our context, we need only note that

$$
\left(\Delta_{i}\right)^{2}=\left[\frac{x_{i}-x_{i-1}}{\Delta s}\right]^{2}(\Delta s)^{2} \approx\left[g^{\prime}\left(s_{i}\right)\right]^{2}(\Delta s)^{2},
$$


and

$$
\Delta_{i+1}-\Delta_{i}=\left[\frac{x_{i+1}-2 x_{i}+x_{i-1}}{\Delta s^{2}}\right](\Delta s)^{2} \approx\left[g^{\prime \prime}\left(s_{i}\right)\right](\Delta s)^{2} .
$$

Thus, the truncation error in (1.6) is now second order in $\Delta s$.

Another method of producing second-order truncation error is the use of implicit-difference schemes for both ordinary and partial differential equations. Such methods have been studied by Osborne [20], Lynch and Rice [18], Doedel [7], Swartz [22], Ciment, Leventhal, and Weinberg [3], among others. We will illustrate these methods with a simple example that is due to Doedel. Instead of a difference approximation like (1.4), an extra degree of freedom is added by looking at equations of the form

$$
\alpha_{i} v_{i-1}+\beta_{i} v_{i}+\gamma_{i} v_{i+1}=f\left(z_{i}\right)
$$

where $\alpha_{i}, \beta_{i}, \gamma_{i}$, and $z_{i}$ are to be determined. Although the precise formulae are tedious to display, we note that if $\alpha_{i}, \beta_{i}$, and $\gamma_{i}$ correspond to the second divided difference and $z_{i}=\left(x_{i-1}+x_{i}+x_{i+1}\right) / 3$, then (1.8) approximates $y^{\prime \prime}=f(x)$ with truncation error that is $O\left(\Delta^{2}\right)$ for uniform and nonuniform meshes.

We show in this paper that, for many common difference schemes, the accuracy is second-order in spite of first-order truncation error. The standard proof, although useful in its proper context, is inadequate to handle nonuniform meshes. We will develop new tools that are applicable to both uniform and nonuniform meshes. As a motivation, consider writing (1.1) as a pair of first-order equations,

$$
\begin{gathered}
y_{1}^{\prime}=y_{2}, \\
y_{2}^{\prime}=-a(x) y_{2}-b(x) y_{1}+f(x),
\end{gathered}
$$

with appropriate boundary conditions. Keller [12] and Keller and White [13] have shown that centered-difference approximations of $(1.9 \mathrm{a}, \mathrm{b})$ are second-order accurate under mild restrictions on the mesh. This approach has the additional advantages that its asymptotic error expansion proceeds in powers of $\left(\Delta_{i}\right)^{2}$ and that approximations to the derivative are just as accurate as those to the solution itself.

In Section 2, we begin our study by looking more closely at the solution of $(1.9 \mathrm{a}, \mathrm{b})$ by centered differences. In the resulting linear system of equations, the unknowns $Y_{2}$ can be eliminated in such a way as to yield a compact-as-possible difference scheme for the unknowns $Y_{1}$. Like (1.6), the truncation error is first-order. However, we know that the accuracy is second-order because it is also the solution of the system $(1.9 \mathrm{a}, \mathrm{b})$. The reduction procedure is examined to illuminate this apparent paradox. A simple result is proved that provides the basis for the remainder of the paper.

In Section 3, we prove that many difference approximations of (1.1) and (1.2a,b) yield second-order accurate solutions, even though their truncation errors are formally first-order on general nonuniform meshes. As an example, we look at (1.4) and show that no new requirements on the mesh, $X$, are necessary to ensure second-order accuracy. Several numerical examples will be given to illustrate this result. In Section 4, these results are extended to quasilinear problems. Section 5 examines these problems for cell-centered difference schemes, and we will discover 
that these schemes are not nearly as forgiving on nonuniform meshes as are vertex-centered methods. Section 6 contains some concluding remarks.

This work, although approached from a completely different direction, is, in part, a rediscovery of the results of Tikhonov and Samarskii [23], whose paper seems to be little appreciated. We are indebted to David Levermore [17] for calling it to our attention. A related approach, generalized to compact schemes for nonselfadjoint or higher-order equations, invokes summation by parts-see, e.g., Kreiss, Manteuffel, Swartz, Wendroff, and White [16]. The work of Grigorieff [9] addresses similar issues. A Spijker norm is used and in this weaker norm, the truncation error is second-order. The difficulty in this approach comes in proving stability. The proofs presented in our paper are elementary and are motivated through the section on first-order systems. The techniques employed here have application to a wide variety of problems not previously addressed.

2. First-Order Systems of Equations. As we noted in Section 1, the differential equation (1.1) can be rewritten as a pair of first-order differential equations and approximated by centered differences. This system will have second-order truncation error, and thus second-order accuracy, on a nonuniform mesh. In order to examine this procedure in detail, it will be sufficient to consider the simple problem

$$
y^{\prime \prime}=f(x) \text {. }
$$

One equivalent first-order system is given by the pair of equations

$$
\begin{aligned}
& y_{1}^{\prime}(x)=y_{2}(x), \\
& y_{2}^{\prime}(x)=f(x),
\end{aligned}
$$

where $y_{1}(x)=y(x)$ and $y_{2}(x)=y^{\prime}(x)$. Keller [12] has shown that it is easy to construct a second-order accurate solution of $(2.2 \mathrm{a}, \mathrm{b})$ using centered-difference approximations,

$$
\begin{gathered}
\frac{1}{\Delta_{i}}\left[v_{i}-v_{i-1}\right]=\frac{1}{2}\left[w_{i}+w_{i-1}\right], \quad i=1, \ldots, N ; \\
\frac{1}{\Delta_{i}}\left[w_{i}-w_{i-1}\right]=f\left(x_{i-1 / 2}\right) \equiv(F)_{i}, \quad i=1, \ldots, N .
\end{gathered}
$$

We may rewrite these equations by defining an $(N \times N+1)$-difference matrix

$$
D_{0} \equiv\left[\begin{array}{cccc}
-\frac{1}{\Delta_{1}} & \frac{1}{\Delta_{1}} & & \\
-\frac{1}{\Delta_{2}} & \frac{1}{\Delta_{2}} & \\
& & & \ddots
\end{array}\right]
$$

and an $(N \times N+1)$-average matrix

$$
A_{0} \equiv\left[\begin{array}{cccc}
\frac{1}{2} & \frac{1}{2} & & \\
& \frac{1}{2} & \frac{1}{2} & \\
& & & \ddots
\end{array}\right] .
$$


With these definitions, Eqs. $(2.3 \mathrm{a}, \mathrm{b})$ become

$$
\begin{gathered}
D_{0} V=A_{0} W, \\
D_{0} W=F .
\end{gathered}
$$

For simplicity, consider Dirichlet boundary conditions appended to (2.1); the discrete approximations are given by

$$
v_{0}=b_{02}, \quad v_{N}=b_{12} \text {. }
$$

Equations $(2.5 \mathrm{a}, \mathrm{b})$ and $(2.6)$ form a system of $(2 N+2)$ equations involving the $(2 N+2)$ unknowns, $V$ and $W$.

Keller [12] proved the following theorem specialized to our example.

THEOREM 2.1. Let $f(x) \in C^{2}(a, b)$; then for all quasi-uniform meshes,

$$
\Delta_{\max } \leqslant \lambda \Delta_{\min },
$$

with $\Delta_{\max } \leqslant \Delta_{0}, \Delta_{0}$ sufficiently small, a unique solution of the discrete boundary value problem $(2.5 \mathrm{a}, \mathrm{b})$ and $(2.6)$ exists and has the property that

$$
\left|y_{1}\left(x_{i}\right)-v_{i}\right| \leqslant C \Delta_{\max }^{2} .
$$

Proof. The proof is through the usual stability, consistency arguments. The truncation error for $(2.3 \mathrm{a}, \mathrm{b})$ is found in the usual way by replacing $v_{i}$ and $w_{i}$ in $(2.3 \mathrm{a}, \mathrm{b})$ with $y_{1}\left(x_{i}\right)$ and $y_{2}\left(x_{i}\right)$, respectively. In the form of $(2.5 \mathrm{a}, \mathrm{b})$, we have

$$
\begin{gathered}
D_{0} Y_{1}=A_{0} Y_{2}+T_{1}, \\
D_{0} Y_{2}=F+T_{2},
\end{gathered}
$$

where a liberal dose of Taylor's series yields

$$
\begin{aligned}
& \left(T_{1}\right)_{i}=-\frac{1}{12} \Delta_{i}^{2} y_{1}^{\prime \prime \prime}\left(x_{i-1 / 2}\right)+O\left(\Delta^{3}\right), \\
& \left(T_{2}\right)_{i}=-\frac{1}{24} \Delta_{i}^{2} y_{1}^{\prime \prime \prime}\left(x_{i-1 / 2}\right)+O\left(\Delta^{3}\right) .
\end{aligned}
$$

Stability will now yield Theorem 2.1 .

However, we can reduce the $(2 N+2)$ equations $(2.5 \mathrm{a}, \mathrm{b})$ and $(2.6)$ to $(N+1)$ equations by eliminating $W$, thus deriving a difference approximation to the original second-order equation, (2.1). If the reduction is done carefully, the new difference scheme will be a three-point scheme and the boundary conditions will remain unaffected. To accomplish this, we define another difference matrix,

$$
D_{1} \equiv\left[\begin{array}{ccc}
-\frac{2}{\Delta_{1}+\Delta_{2}} & \frac{2}{\Delta_{1}+\Delta_{2}} & \\
& -\frac{2}{\Delta_{2}+\Delta_{3}} & \frac{2}{\Delta_{2}+\Delta_{3}} \\
& & \ddots
\end{array}\right],
$$

and another average matrix,

$$
A_{1} \equiv\left[\begin{array}{ccc}
\frac{\Delta_{1}}{\Delta_{1}+\Delta_{2}} & \frac{\Delta_{2}}{\Delta_{1}+\Delta_{2}} & \\
& \frac{\Delta_{2}}{\Delta_{2}+\Delta_{3}} & \frac{\Delta_{3}}{\Delta_{2}+\Delta_{3}} \\
& & \ddots
\end{array}\right],
$$


where both matrices are $(N-1) \times N$. Recalling $(2.4 \mathrm{a}, \mathrm{b})$, it can be shown that

$$
A_{1} D_{0}=D_{1} A_{0} \text {. }
$$

If we left-multiply (2.5a) by $D_{1}$, left-multiply (2.5b) by $A_{1}$, envoke (2.12), and add the equations, we have

$$
D_{1} D_{0} V=A_{1} F
$$

where $D_{1} D_{0} \equiv D_{2}$ is the second divided-difference operator as defined in (1.3a).

The truncation error associated with (2.13) can be derived in two equivalent ways. Replacing $V$ in (2.13) by the exact solution, $Y_{1}$, in the usual fashion, we get

$$
D_{1} D_{0} Y_{1}=A_{1} F+T \text {, }
$$

where a Taylor's series expansion yields

$$
(T)_{i}=-\frac{1}{6}\left(\Delta_{i+1}-\Delta_{i}\right) y^{\prime \prime \prime}\left(x_{i}\right)+O\left(\Delta^{2}\right) .
$$

However, we may also derive the truncation error by repeating the reduction shown in Eqs. (2.12) and (2.13), and eliminating $Y_{2}$ from (2.9a,b). This procedure yields

$$
D_{1} D_{0} Y_{1}=A_{1} F+D_{1} T_{1}+A_{1} T_{2} \text {. }
$$

Thus, the truncation error, $T$, in (2.14) has the representation

$$
T=D_{1} T_{1}+A_{1} T_{2} .
$$

Recalling the expressions $(2.10 \mathrm{a}, \mathrm{b})$, we see that $A_{1} T_{2}$ is $O\left(\Delta^{2}\right)$ because $A_{1}$ is an averaging matrix. The term $D_{1} T_{1}$ provides the troublesome $\left(\Delta_{i+1}-\Delta_{i}\right)$-term in (2.15).

The error equation is found by subtracting (2.13) from (2.16), which yields

$$
D_{1} D_{0} E=D_{1} T_{1}+A_{1} T_{2},
$$

where $(E)_{i} \equiv y\left(x_{i}\right)-v_{i}$. Assuming stability of the difference scheme and ignoring boundary conditions, Eq. (2.17) yields an error estimate

$$
\|E\|_{\infty} \leqslant C\left\|D_{1} T_{1}\right\|_{\infty}+C\left\|A_{1} T_{2}\right\|_{\infty} \text {. }
$$

Recalling (2.10a,b), we have

$$
\begin{gathered}
\left\|D_{1} T_{1}\right\|_{\infty} \leqslant C \max _{i}\left|\Delta_{i+1}-\Delta_{i}\right|=O(\Delta), \\
\left\|A_{1} T_{2}\right\|_{\infty} \leqslant C \max _{i}\left|\Delta_{i}^{2}\right|=O\left(\Delta^{2}\right) .
\end{gathered}
$$

From (2.17) we see that the error can be divided into two terms, $E=E_{1}+E_{2}$, such that

$$
\begin{aligned}
& D_{1} D_{0} E_{1}=D_{1} T_{1}, \\
& D_{1} D_{0} E_{2}=A_{1} T_{2} .
\end{aligned}
$$

The usual arguments applied to $(2.19 \mathrm{~b})$ yield second-order accuracy. However, these same techniques applied to (2.19a), using (2.18a), give a poor approximation, i.e., $E_{1}$ is $O(\Delta)$, yet we know from Theorem 2.1 that the scheme is second-order accurate. A careful accounting of $E_{1}$ must produce an estimate similar to (2.8). The following lemma examines the solution of (2.19a) and provides a key to many of the results in the following sections. 
LeMma 2.2. On any mesh, define an $(N+1)$-vector, $E_{1}$, by

$$
\left(E_{1}\right)_{0}=0
$$

$$
\left(E_{1}\right)_{i}=\sum_{j=1}^{i} \Delta_{j}\left(T_{1}\right)_{j}, \quad i=1, \ldots, N .
$$

Then $E_{1}$ is a solution of (2.19a). Further,

$$
\begin{gathered}
\left\|E_{1}\right\|_{\infty} \leqslant\left\|T_{1}\right\|_{\infty}, \\
\left\|D_{0} E_{1}\right\|_{\infty}=\left\|T_{1}\right\|_{\infty} .
\end{gathered}
$$

Proof. Let us write (2.19a) as

$$
D_{1}\left(D_{0} E_{1}-T_{1}\right)=0
$$

The quantity in parentheses must be in the null space of $D_{1}$, that is, a constant vector that we shall denote as $\lambda \mathbf{1}$. The vector $E_{1}$ must satisfy

$$
D_{0} E_{1}=T_{1}+\lambda \mathbf{1} \text {. }
$$

The components of this equation are

$$
\left(E_{1}\right)_{i}=\left(E_{1}\right)_{i-1}+\Delta_{i}\left(T_{1}\right)_{i}+\lambda \Delta_{i}, \quad i=1, \ldots, N-1 .
$$

Summing the right-hand side yields

$$
\left(E_{1}\right)_{i}=\left(E_{1}\right)_{0}+\sum_{j=1}^{i} \Delta_{j}\left(T_{1}\right)_{j}+\lambda x_{i} .
$$

We are only interested in a particular solution of (2.19a) and so shall choose $\left(E_{1}\right)_{0}=\lambda=0$, which yields $(2.20 \mathrm{a}, \mathrm{b})$. The bound $(2.21 \mathrm{a})$ is derived by taking absolute values in (2.20b) to get

$$
\left|\left(E_{1}\right)_{i}\right| \leqslant\left|\sum_{j=1}^{i} \Delta_{j}\right|\left\|T_{1}\right\|_{\infty} \leqslant\left\|T_{1}\right\|_{\infty} .
$$

Equality (2.21b) follows immediately from (2.22) with $\lambda=0$.

In the next section, we show that many truncation error expressions, like (2.15) and (1.6), can be rewritten in the form

$$
T=D_{1} T_{1}+T_{2},
$$

where $T_{1}$ and $T_{2}$ are both second-order. Lemma 2.2 will then provide the means for showing that the solution error is second-order accurate in such cases.

3. Linear Second-Order Equations. In Section 2, we saw that at least one difference scheme approximating the equation

$$
y^{\prime \prime}=f(x)
$$

with Dirichlet boundary conditions yields second-order accurate solutions on nonuniform meshes. In this section, we extend our discussion to linear second-order equations of the form (1.1) and (1.2a,b). Second-order accurate difference schemes for (1.1) can be constructed by forming an associated first-order system, discretizing with centered differences, and carefully eliminating the auxiliary unknowns as described in Section 2. More will be said about this and higher-order equations in a 
subsequent paper. In this paper, we prove a result that will help to identify those three-point difference approximations to $(1.1)$ and $(1.2 \mathrm{a}, \mathrm{b})$ that are second-order accurate.

We use the notation of Section 1, namely,

$$
\begin{gathered}
\left(L_{h} V\right)_{i}=(F)_{i}, \quad i=1, \ldots, N-1, \\
B_{0} V=b_{02}, \\
B_{1} V=b_{12} .
\end{gathered}
$$

The truncation error will be defined by replacing $V$ with $Y$ in (3.1) and (3.2a,b).

Many difference schemes have the property that the truncation error can be divided into two parts,

$$
T=\tilde{T}_{1}+\tilde{T}_{2}
$$

where

$$
\left(\tilde{T}_{1}\right)_{0}=\left(\tilde{T}_{1}\right)_{N}=0 \text {, }
$$

$$
\left(\tilde{T}_{1}\right)_{i}=\left(\Delta_{i+1}-\Delta_{i}\right) p\left(x_{i}\right), \quad i=1, \ldots, N-1,
$$

for some function $p(x) \in C^{1}(0,1)$, and

$$
\left\|\tilde{T}_{2}\right\|_{\infty} \leqslant C \Delta_{\max }^{2}
$$

The next lemma will show that difference schemes of this type may be second-order accurate.

LEMMA 3.1. Let the truncation error for a difference scheme (3.1) and (3.2a,b) satisfy (3.3) and (3.4a,b,c); then

$$
\begin{gathered}
(T)_{0}=\left(\tilde{T}_{2}\right)_{0}, \\
(T)_{N}=\left(\tilde{T}_{2}\right)_{N}, \\
(T)_{i}=\left(D_{1} T_{1}+T_{2}\right)_{i}, \quad i=1, \ldots, N-1,
\end{gathered}
$$

where $T_{1}$ is an $N$-vector, $T_{2}$ is an $(N+1)$-vector, $D_{1}$ is defined in $(2.11 \mathrm{a})$, and

$$
\left\|T_{1}\right\|_{\infty} \leqslant C \Delta_{\max }^{2}, \quad\left\|T_{2}\right\|_{\infty} \leqslant C \Delta_{\max }^{2} .
$$

Proof. From (3.4b), note that

$$
\left(\tilde{T}_{1}\right)_{i}=\frac{\Delta_{i+1}^{2}}{\Delta_{i+1}+\Delta_{i}} p\left(x_{i}\right)-\frac{\Delta_{i}^{2}}{\Delta_{i+1}+\Delta_{i}} p\left(x_{i}\right) .
$$

The function $p(x)$ is differentiable, so we can rewrite this expression as

$$
\begin{aligned}
\left(\tilde{T}_{1}\right)_{i}= & \frac{1}{\Delta_{i+1}+\Delta_{i}}\left[\Delta_{i+1}^{2} p\left(x_{i+1 / 2}\right)-\Delta_{i}^{2} p\left(x_{i-1 / 2}\right)\right] \\
& +\frac{\Delta_{i+1}^{3}}{2\left(\Delta_{i+1}+\Delta_{i}\right)} p^{\prime}\left(\xi_{1}\right)+\frac{\Delta_{i}^{3}}{2\left(\Delta_{i+1}+\Delta_{i}\right)} p^{\prime}\left(\xi_{2}\right),
\end{aligned}
$$

for some $\xi_{1}$ and $\xi_{2} \in\left(x_{i-1}, x_{i+1}\right)$. Now, identify

$$
\left(T_{1}\right)_{i} \equiv \frac{1}{2} \Delta_{i}^{2} p\left(x_{i-1 / 2}\right), \quad i=1, \ldots, N,
$$


and

$$
\begin{aligned}
\left(T_{2}\right)_{i} \equiv\left(\tilde{T}_{2}\right)_{i}+\frac{1}{2\left(\Delta_{i+1}+\Delta_{i}\right)}\left[\Delta_{i+1}^{3} p^{\prime}\left(\xi_{1}\right)+\Delta_{i}^{3} p^{\prime}\left(\xi_{2}\right)\right] & \\
& i=1, \ldots, N-1 .
\end{aligned}
$$

With these definitions, we have proved $(3.5 \mathrm{a}, \mathrm{b}, \mathrm{c})$. If we recall that $p(x)$ is continuously differentiable on $[0,1]$, then (3.6) implies that

$$
\left\|T_{1}\right\|_{\infty} \leqslant \max _{[0,1]}\{|p(x)|\} \Delta_{\max }^{2}
$$

with no constraints on the mesh. To complete the definition of $T_{2}$, we take

$$
(T)_{0} \equiv\left(\tilde{T}_{2}\right)_{0} \equiv\left(T_{2}\right)_{0}, \quad(T)_{N} \equiv\left(\tilde{T}_{2}\right)_{N} \equiv\left(T_{2}\right)_{N}
$$

A bound for (3.7) independent of the mesh is derived by noting that

$$
\frac{1}{2\left(\Delta_{i+1}+\Delta_{i}\right)}\left[\Delta_{i+1}^{3} p^{\prime}\left(\xi_{1}\right)+\Delta_{i}^{3} p^{\prime}\left(\xi_{2}\right)\right] \leqslant \max _{[0,1]}\left\{\left|p^{\prime}(x)\right|\right\} \Delta_{\max }^{2} .
$$

Thus, again without any constraints on the mesh, we have

$$
\left\|T_{2}\right\|_{\infty} \leqslant C \Delta_{\max }^{2}
$$

The hypothesis of Lemma 3.1, Eqs. (3.4a,b,c), is satisfied by many difference schemes. The second divided difference applied to $y^{\prime \prime}$ yields

$$
(T)_{i}=\frac{1}{3}\left(\Delta_{i+1}-\Delta_{i}\right) y^{\prime \prime \prime}+O\left(\Delta^{2}\right), \quad i=1, \ldots, N-1 .
$$

Consider several reasonable methods of approximating $y^{\prime}$. If we take the difference of values at $x_{i-1}$ and $x_{i+1}$, we have

$$
\left[-\frac{1}{\Delta_{i+1}+\Delta_{i}}, 0, \frac{1}{\Delta_{i+1}+\Delta_{i}}\right]\left[\begin{array}{c}
y_{i-1} \\
y_{i} \\
y_{i+1}
\end{array}\right]=y^{\prime}+\frac{1}{2}\left(\Delta_{i+1}-\Delta_{i}\right) y^{\prime \prime}+O\left(\Delta^{2}\right) \text {. }
$$

If we average function values on the left and right subintervals and take the difference of the averages, we have

$$
\left[-\frac{1}{2 \Delta_{i}}, \frac{\Delta_{i+1}-\Delta_{i}}{2 \Delta_{i} \Delta_{i+1}}, \frac{1}{2 \Delta_{i+1}}\right]\left[\begin{array}{c}
y_{i-1} \\
y_{i} \\
y_{i+1}
\end{array}\right]=y^{\prime}+\frac{1}{4}\left(\Delta_{i+1}-\Delta_{i}\right) y^{\prime \prime}+O\left(\Delta^{2}\right) \text {. }
$$

Recall that the approximation (1.3b) used in Pearson [21] is

$$
\left[\frac{-\Delta_{i+1}}{\Delta_{i}\left(\Delta_{i+1}+\Delta_{i}\right)}, \frac{\Delta_{i+1}-\Delta_{i}}{\Delta_{i+1} \Delta_{i}}, \frac{\Delta_{i}}{\Delta_{i+1}\left(\Delta_{i+1}+\Delta_{i}\right)}\right]\left[\begin{array}{c}
y_{i-1} \\
y_{i} \\
y_{i+1}
\end{array}\right]=y^{\prime}+O\left(\Delta^{2}\right) \text {. }
$$

Each of the functions on the right-hand sides of these truncation errors is evaluated at $x_{i}$. Note that each scheme has a truncation error of the form (3.3), and that each of these approximations is the same on a uniform mesh. With Lemmas 2.2 and 3.1 in hand, we now examine the question of second-order accuracy for more general difference schemes.

THEOREM 3.2. Let the difference scheme (3.1) and (3.2a,b) have the following properties:

(i) the difference scheme is stable for all meshes in some class $M$ with $\Delta_{\max } \leqslant \Delta_{0}$; 
(ii) $L_{h}=D_{2}+L_{1}$, where $D_{2}$ is the second divided difference; and

(iii) the truncation errors satisfy the hypothesis of Lemma 3.1 (Eqs. (3.3) and $(3.4 \mathrm{a}, \mathrm{b}, \mathrm{c}))$.

Then, the error at the mesh points is bounded by

$$
\|E\|_{\infty} \leqslant C\left[\left\|T_{1}\right\|_{\infty}+\left\|T_{2}\right\|_{\infty}+\left\|R E_{1}\right\|_{\infty}\right],
$$

where $E_{1}$ is defined as in Lemma 2.2, $T_{1}$ and $T_{2}$ are defined in Lemma 3.1, and $R$ is an $(N+1) \times(N+1)$-matrix,

$$
\begin{gathered}
(R V)_{0}=\left(B_{0} V\right)_{0}, \\
(R V)_{i}=\left(L_{1} V\right)_{i}, \quad i=1, \ldots, N-1, \\
(R V)_{N}=\left(B_{1} V\right)_{N} .
\end{gathered}
$$

In other words, $R$ is the difference operator without the second divided difference.

Proof. The equations for the pointwise error, $E$, are

$$
\begin{gathered}
\left(B_{0} E\right)_{0}=(T)_{0}, \\
\left(\left[D_{2}+L_{1}\right] E\right)_{i}=(T)_{i}, \quad i=1, \ldots, N-1, \\
\left(B_{1} E\right)_{N}=(T)_{N} .
\end{gathered}
$$

Recalling that $D_{2}=D_{1} D_{0}$, and using Lemma 3.1, we have

$$
\begin{aligned}
& \left(B_{0} E\right)_{0}=\left(T_{2}\right)_{0}, \\
\left(\left[D_{1} D_{0}+L_{1}\right] E\right)_{i}= & \left(D_{1} T_{1}\right)_{i}+\left(T_{2}\right)_{i}, \quad i=1, \ldots, N-1, \\
& \left(B_{1} E\right)_{N}=\left(T_{2}\right)_{N} .
\end{aligned}
$$

Let us define

$$
E=E_{1}+E_{2} \text {, }
$$

where $E_{1}$ is as given in $(2.20 \mathrm{a}, \mathrm{b})$. Then, because $E_{1}$ satisfies $D_{1} D_{0} E_{1}=D_{1} T_{1}$, we have

$$
\begin{gathered}
\left(B_{0} E_{2}\right)_{0}=\left(T_{2}\right)_{0}-\left(R E_{1}\right)_{0}, \\
\left(L_{h} E_{2}\right)_{i}=\left(T_{2}\right)_{i}-\left(R E_{1}\right)_{i}, \quad i=1, \ldots, N-1, \\
\left(B_{N} E_{2}\right)_{N}=\left(T_{2}\right)_{N}-\left(R E_{1}\right)_{N} .
\end{gathered}
$$

By hypothesis, the difference scheme is stable, which yields the estimate

$$
\left\|E_{2}\right\|_{\infty} \leqslant C\left(\left\|T_{2}\right\|_{\infty}+\left\|R E_{1}\right\|_{\infty}\right) \text {. }
$$

Combining this with the bound for $E_{1}$ found in Lemma 2.2 yields

$$
\|E\|_{\infty} \leqslant\left\|E_{1}\right\|_{\infty}+\left\|E_{2}\right\|_{\infty} \leqslant C\left(\left\|T_{1}\right\|_{\infty}+\left\|T_{2}\right\|_{\infty}+\left\|R E_{1}\right\|_{\infty}\right) \text {. }
$$

The shortcoming of this general result is that we do not have bounds on the last term in (3.8). Before we prove a more practical result, let us examine what this term represents. In most schemes, $L_{1}$ will be a consistent approximation to a first-order differential operator, say

$$
g(x) \frac{d}{d x}+h(x)
$$


and $B_{0}, B_{1}$ will be consistent approximations to the boundary conditions

$$
\begin{aligned}
& b_{01} \frac{d}{d x}+b_{00}, \\
& b_{11} \frac{d}{d x}+b_{10} .
\end{aligned}
$$

Taking liberties with notation, the error $E_{1}$, recall $(2.20 \mathrm{a}, \mathrm{b})$, can be written as

$$
E_{1} \equiv \int_{0}^{x} T_{1} d x
$$

Applying $(3.10 \mathrm{a}, \mathrm{b}, \mathrm{c})$ to this expression yields

$$
\begin{gathered}
g(x) T_{1}+h(x) \int_{0}^{x} T_{1} d x, \\
b_{01}\left(T_{1}\right)_{0} \\
b_{11}\left(T_{1}\right)_{N}+b_{10} \int_{0}^{1} T_{1} d x .
\end{gathered}
$$

Clearly, all terms can be bounded by $C\left\|T_{1}\right\|_{\infty}$. Tikhonov and Samarskii [23] remark that their result illustrates the global nature of the error. Expressions $(3.11 \mathrm{a}, \mathrm{b}, \mathrm{c})$ show that there is both a global and a local component to the error if $g(x) \neq 0$.

Corollary 3.3 will show that the heuristic argument developed above is correct.

COROLlaRY 3.3. Let the discrete boundary value problem satisfy all the conditions of Theorem 3.2. Further, let $B_{0}, L_{1}, B_{1}$ be consistent, three-point difference approximations:

$$
\begin{gathered}
\left(B_{0} V\right)_{0}=\alpha_{0} v_{0}+\beta_{0} v_{1}+\gamma_{0} v_{2}, \\
\left(L_{1} V\right)_{i}=\alpha_{i} v_{i-1}+\beta_{i} v_{i}+\gamma_{i} v_{i+1}, \quad i=1, \ldots, N-1, \\
\left(B_{1} V\right)_{N}=\alpha_{N} v_{N-2}+\beta_{N} v_{N-1}+\gamma_{N} v_{N}
\end{gathered}
$$

to $b_{00}+b_{01} d / d x, a(x) d / d x+b(x)$, and $b_{10}+b_{11} d / d x$, respectively; let

$$
\max _{i}\left\{\left|\gamma_{i} \Delta_{i+1}\right|,\left|\alpha_{i} \Delta_{i}\right|\right\} \leqslant C, \quad i=1, \ldots, N-1
$$

and let $\max \left\{\left|\gamma_{0} \Delta_{2}\right|,\left|\alpha_{0} \Delta_{1}\right|,\left|\gamma_{N} \Delta_{N}\right|,\left|\alpha_{N} \Delta_{N-1}\right|\right\} \leqslant C$. Then,

$$
\|Y-V\|_{\infty} \leqslant C \Delta_{\max }^{2} \text {. }
$$

Proof. Theorem 3.2 and Lemma 3.1 yield the result

$$
\|E\|_{\infty}=\|Y-V\|_{\infty} \leqslant C \Delta_{\max }^{2}+C\left\|R E_{1}\right\|_{\infty},
$$

so it is this last term we need to estimate. Looking at (3.12b), we have

$$
\left(R E_{1}\right)_{i}=\left(\alpha_{i}+\beta_{i}+\gamma_{i}\right)\left(E_{1}\right)_{i}+\left[\gamma_{i} \Delta_{i+1}\left(T_{1}\right)_{i+1}-\alpha_{i} \Delta_{i}\left(T_{1}\right)_{i}\right],
$$

and, consequently,

$$
\left|\left(R E_{1}\right)_{i}\right| \leqslant\left|\alpha_{i}+\beta_{i}+\gamma_{i}\right|\left\|E_{1}\right\|_{\infty}+2 \max _{i}\left\{\left|\gamma_{i} \Delta_{i+1}\right|,\left|\alpha_{i} \Delta_{i}\right|\right\}\left\|T_{1}\right\|_{\infty} .
$$

Clearly, consistency requires that $\left|\left(\alpha_{i}+\beta_{i}+\gamma_{i}\right)\right| \leqslant C$ for all meshes with $\Delta_{\max } \leqslant \Delta_{0}$. From Lemma 2.2, we have

$$
\left\|E_{1}\right\|_{\infty} \leqslant C\left\|T_{1}\right\|_{\infty}
$$


thus,

$$
\left|\left(R E_{1}\right)_{i}\right| \leqslant C\left\|T_{1}\right\|_{\infty} .
$$

The boundary conditions $(3.12 \mathrm{a}, \mathrm{c})$ are handled in a similar fashion. Coupled with Lemma 3.1, we now have

$$
\|E\|_{\infty} \leqslant C \Delta_{\max }^{2} .
$$

Condition (3.13) is, at worst, a constraint on the local mesh ratio. However, if we look at the difference scheme (1.4), we note that

$$
\left|\gamma_{i} \Delta_{i+1}\right|=\left|a\left(x_{i}\right) \frac{\Delta_{i}}{\Delta_{i+1}+\Delta_{i}}\right| \leqslant \max _{[0,1]}\{|a(x)|\},
$$

and

$$
\left|\alpha_{i} \Delta_{i}\right|=\left|a\left(x_{i}\right) \frac{\Delta_{i+1}}{\Delta_{i+1}+\Delta_{i}}\right| \leqslant \max _{[0,1]}\{|a(x)|\},
$$

so that (3.13) is satisfied with no mesh restrictions at all. For the boundary conditions, we might use the second-order accurate scheme at $x=1$. This yields

$$
\begin{aligned}
b_{10} v_{N}+b_{11} & \frac{\Delta_{N}}{\Delta_{N-1}\left(\Delta_{N}+\Delta_{N-1}\right)} v_{N-2} \\
& \left.\quad-\frac{\Delta_{N}+\Delta_{N-1}}{\Delta_{N} \Delta_{N-1}} v_{N-1}+\frac{2 \Delta_{N}+\Delta_{N-1}}{\Delta_{N}\left(\Delta_{N}+\Delta_{N-1}\right)} v_{N}\right]=b_{12} .
\end{aligned}
$$

Thus, we have

$$
\begin{gathered}
\left|\gamma_{N} \Delta_{N}\right| \leqslant\left|b_{10}\right| \Delta_{N}+\left|b_{11}\right| \frac{2 \Delta_{N}+\Delta_{N-1}}{\Delta_{N}+\Delta_{N-1}} \leqslant C, \\
\left|\alpha_{N} \Delta_{N-1}\right| \leqslant\left|b_{11}\right| \frac{\Delta_{N}}{\Delta_{N}+\Delta_{N-1}} \leqslant C .
\end{gathered}
$$

This also does not require any special restrictions on the mesh. The same thing will be true for this boundary condition applied at $x=0$. Thus, we have shown that Pearson's scheme, if stable for a class of meshes with $\max _{i}\left\{\Delta_{i}\right\} \leqslant \Delta_{0}$, will be second-order accurate for those same meshes. The same is true for many other commonly used difference schemes.

4. Quasilinear, Second-Order Equations. In this section, we extend the basic result for linear boundary value problems to the solution of quasilinear boundary value problems. These will be of the form

$$
\begin{aligned}
y^{\prime \prime}= & f\left(y^{\prime}, y, x\right), \quad x \in(0,1), \\
& b_{0}\left(y(0), y^{\prime}(0)\right)=0, \\
& b_{1}\left(y(1), y^{\prime}(1)\right)=0 .
\end{aligned}
$$

Throughout we will assume that (4.1) and (4.2a,b) have an isolated solution, see Keller and White [13], $y(x) \in C^{4}[0,1]$. The difference equations approximating this boundary value problem will be denoted by

$$
\left(N_{h}(V)\right)_{i}=0, \quad i=1, \ldots, N-1 ;
$$




$$
\begin{aligned}
& B_{0}(V)=0 ; \\
& B_{1}(V)=0 .
\end{aligned}
$$

Together, we write these equations as

$$
N(V)=\left[\begin{array}{l}
B_{0}(V) \\
N_{h}(V) \\
B_{1}(V)
\end{array}\right],
$$

and once again assuming that $y^{\prime \prime}$ is approximated by the second divided difference, let

$$
R(V)=N(V)-\left[\begin{array}{c}
0 \\
D_{2} V \\
0
\end{array}\right]=\left[\begin{array}{c}
B_{0}(V) \\
N_{1}(V) \\
B_{1}(V)
\end{array}\right] .
$$

The truncation error, $T$, will be defined in the usual way,

$$
N(Y)=T .
$$

Stability of the difference scheme for quasilinear problems is a local phenomenon (cf. Keller and White [13], Doedel [8]). We will define a cylinder around the solution by

$$
S_{1}(Y, \rho)=\left\{V \mid\|V-Y\|_{\infty} \leqslant \rho,\left\|D_{0} V-Y^{\prime}\right\|_{\infty} \leqslant \rho\right\},
$$

where $D_{0} V$ is an $N$-vector consisting of first divided differences and $\left(Y^{\prime}\right)_{i}=$ $y^{\prime}\left(x_{i-1 / 2}\right), i=1, \ldots, N$. Stability of the difference scheme (4.3) and $(4.4 \mathrm{a}, \mathrm{b})$ is taken to mean there exists $C$, and some $\rho>0$, such that

$$
\|V-W\| \leqslant C\|N(V)-N(W)\|,
$$

for every $V, W \in S_{1}(Y, \rho)$. With these definitions, we have the following generalization of Theorem 3.2 .

THEOREM 4.1. Let the difference scheme (4.3) and (4.4a,b) have the following properties:

(i) the difference scheme is stable, in the sense of (4.7), for all meshes in some class $M$ with $\Delta_{\max } \leqslant \Delta_{0}$;

(ii) $N_{h}(V)=D_{2} V+N_{1}(V)$, where $D_{2}$ is the second divided difference; and

(iii) the truncation errors satisfy Eqs. (3.3) and (3.4a,b,c).

Then, if $N(V)=0, V \in S_{1}(Y, \rho)$, the error at the mesh points satisfies

$$
\|E\|_{\infty} \leqslant C\left[\left\|T_{1}\right\|_{\infty}+\left\|T_{2}\right\|_{\infty}+\left\|R\left(Y-E_{1}\right)-R(Y)\right\|_{\infty}\right],
$$

where, as before, $E_{1}$ is defined in Lemma 2.2, $T_{1}$ and $T_{2}$ are defined in Lemma 3.1, and $R$ is defined in (4.5b).

Proof. Let us write the error, $E$, in the following way:

$$
E=E_{1}+E_{2} \text {. }
$$

Since $E=Y-V$, we have the expression

$$
E_{2}=\left(Y-E_{1}\right)-V \text {. }
$$


By assumption, $V \in S_{1}(Y, \rho)$, and by Lemma 2.2, we can choose $\Delta_{0}$ sufficiently small so that $Y-E_{1} \in S_{1}(Y, \rho)$. Applying the stability bound (4.7) yields

$$
\left\|E_{2}\right\|_{\infty} \leqslant C\left\|N\left(Y-E_{1}\right)\right\|_{\infty} .
$$

Because $N(Y)=T$, where $T$ is the truncation error, we may write

$$
\left\|E_{2}\right\|_{\infty} \leqslant C\left\|N\left(Y-E_{1}\right)-N(Y)+T\right\|_{\infty} .
$$

Let us denote the right-hand side as

$$
F=N\left(Y-E_{1}\right)-N(Y)+T .
$$

Consider the components of this equation. We have for the boundary conditions

$$
\begin{aligned}
& (F)_{0}=B_{0}\left(Y-E_{1}\right)-B_{0}(Y)+\left(T_{2}\right)_{0}, \\
& (F)_{N}=B_{1}\left(Y-E_{1}\right)-B_{1}(Y)+\left(T_{2}\right)_{N},
\end{aligned}
$$

and for the differential equation, we have

$$
(F)_{i}=\left(D_{2} Y-D_{2} E_{1}+N_{1}\left(Y-E_{1}\right)-D_{2} Y-N_{1}(Y)\right)_{i}+D_{1}\left(T_{1}\right)_{i}+\left(T_{2}\right)_{i}
$$

for $i=1, \ldots, N-1$. Recalling the definition of $E_{1}$, this becomes

$$
(F)_{i}=\left(T_{2}\right)_{i}+\left(N_{1}\left(Y-E_{1}\right)-N_{1}(Y)\right)_{i} .
$$

Combining (4.9a,b,c), we have

$$
\left\|E_{2}\right\|_{\infty} \leqslant C\left[\left\|T_{2}\right\|_{\infty}+\left\|R\left(Y-E_{1}\right)-R(Y)\right\|_{\infty}\right] .
$$

From Lemma 2.2, we know that $\left\|E_{1}\right\|_{\infty} \leqslant C\left\|T_{1}\right\|_{\infty}$, which yields the result:

$$
\|E\|_{\infty} \leqslant C\left[\left\|T_{1}\right\|_{\infty}+\left\|T_{2}\right\|_{\infty}+\left\|R\left(Y-E_{1}\right)-R(Y)\right\|_{\infty}\right] \text {. }
$$

This error estimate reduces to (3.8) if $R$ is linear. However, an extra hypothesis is required for Theorem 4.1 because it is a local result; that is, we assume that $V \in S_{1}(Y, \rho)$. This condition can be removed in many instances by further restricting the maximum allowable mesh size, but we will not duplicate the work of Keller [14], Doedel [8], Keller and White [13], and others here.

A result very similar to Corollary 3.3 requires that we look closely at the linearized difference equations (4.3) and (4.4a,b). These linear operators are given below:

$$
\begin{gathered}
D_{2} V+\frac{\partial N_{1}}{\partial V}(Y) V, \\
\frac{\partial B_{0}}{\partial V}(Y) V, \\
\frac{\partial B_{1}}{\partial V}(Y) V,
\end{gathered}
$$

where the $i, j$ th element of $\partial N_{1} / \partial V$ is $\partial\left(N_{1}\right)_{i} / \partial v_{j}$.

COROLlaRY 4.2. Let the discrete boundary value problem satisfy the conditions of Theorem 4.1. Further, let the linearized, discrete operators $\partial R(Y) / \partial V$ be consistent, three-point difference approximations to the first-order portion of the linearized boundary value problem; let $\partial R / \partial V$ be uniformly continuous in $S_{1}(\rho, Y)$; let

$$
\max _{i}\left\{\left|\frac{\partial(R)_{i}}{\partial v_{i+1}} \Delta_{i+1}\right|,\left|\frac{\partial(R)_{i}}{\partial v_{i-1}} \Delta_{i}\right|\right\} \leqslant C, \quad i=1, \ldots, N-1
$$


and let

$$
\left\{\left|\frac{\partial B_{0}}{\partial v_{2}} \Delta_{2}\right|,\left|\frac{\partial B_{0}}{\partial v_{0}} \Delta_{1}\right|,\left|\frac{\partial B_{1}}{\partial v_{N}} \Delta_{N}\right|,\left|\frac{\partial B_{1}}{\partial v_{N-2}} \Delta_{N-1}\right|\right\} \leqslant C
$$

Then,

$$
\|Y-V\|_{\infty} \leqslant C \Delta_{\max }^{2}
$$

Proof. The proof proceeds in very much the same way as Corollary 3.3. Theorem 4.1 and Lemma 3.1 yield the result:

$$
\|E\|_{\infty} \leqslant C \Delta_{\max }^{2}+C\left\|R\left(Y-E_{1}\right)-R(Y)\right\|_{\infty} .
$$

To estimate the last term on the right-hand side of (4.13), we write

$$
R\left(Y-E_{1}\right)-R(Y)=-\int_{0}^{1} \frac{\partial R}{\partial V}\left(Y-s E_{1}\right) E_{1} d s
$$

Now, we define

$$
\left[\alpha_{i}(Z), \beta_{i}(Z), \gamma_{i}(Z)\right] \equiv\left[\frac{\partial(R)_{i}}{\partial v_{i-1}}(Z), \frac{\partial(R)_{i}}{\partial v_{i}}(Z), \frac{\partial(R)_{i}}{\partial v_{i+1}}(Z)\right]
$$

so that we have

$$
\begin{aligned}
& \left(R\left(Y-E_{1}\right)-R(Y)\right)_{i} \\
& \quad=-\int_{0}^{1}\left(\alpha_{i}\left(Y-s E_{1}\right)\left(E_{1}\right)_{i-1}+\beta_{i}\left(Y-s E_{1}\right)\left(E_{1}\right)_{i}+\gamma_{i}\left(Y-s E_{1}\right)\left(E_{1}\right)_{i+1}\right) d s
\end{aligned}
$$

for $i=1, \ldots, N-1$. Recalling that $\left(E_{1}\right)_{i}=\sum_{j=0}^{i} \Delta_{j}\left(T_{1}\right)_{j}$, we see that

$$
\begin{aligned}
(R(Y- & \left.\left.E_{1}\right)-R(Y)\right)_{i} \\
= & -\int_{0}^{1}\left(\alpha_{i}\left(Y-s E_{1}\right)+\beta_{i}\left(Y-s E_{1}\right)+\gamma_{i}\left(Y-s E_{1}\right)\right)\left(E_{1}\right)_{i} d s \\
& \quad-\int_{0}^{1}\left(\gamma_{i}\left(Y-s E_{1}\right) \Delta_{i+1}\left(T_{1}\right)_{i+1}-\alpha_{i}\left(Y-s E_{1}\right) \Delta_{i}\left(T_{1}\right)_{i}\right) d s
\end{aligned}
$$

for $i=1, \ldots, N-1$. Because the difference operator (4.14) with $Z=Y$ is a consistent approximation to a first-order differential operator, and because $\partial R(Z) / \partial V$ is uniformly continuous on $S_{1}(Y, \rho)$, we have

$$
\left[\alpha_{i}\left(Y-s E_{1}\right)+\beta_{i}\left(Y-s E_{1}\right)+\gamma_{i}\left(Y-s E_{1}\right)\right] \leqslant C
$$

for all meshes with $\Delta_{0}$ sufficiently small. Likewise, we have assumed that

$$
\max _{i}\left\{\left|\gamma_{i}(Y) \Delta_{i+1}\right|,\left|\alpha_{i}(Y) \Delta_{i}\right|\right\} \leqslant C
$$

so that

$$
\max _{i}\left\{\left|\gamma_{i}\left(Y-s E_{1}\right) \Delta_{i+1}\right|,\left|\alpha_{i}\left(Y-s E_{1}\right) \Delta_{i}\right|\right\} \leqslant C
$$

for all meshes with $\Delta_{0}$ sufficiently small. The boundary conditions can be handled in a similar fashion; thus, from Eq. (4.14), we have

$$
\left\|R\left(Y-E_{1}\right)-R(Y)\right\|_{\infty} \leqslant C\left[\left\|E_{1}\right\|_{\infty}+\left\|T_{1}\right\|_{\infty}\right],
$$


and finally from (4.13), we have

$$
\|E\|_{\infty} \leqslant C \Delta_{\max }^{2}
$$

for all meshes with $\Delta_{0}$ sufficiently small.

These results show that the nonlinearity in the boundary value problem (4.1) and $(4.2 \mathrm{a}, \mathrm{b})$ poses no essential difficulty in extending the results of Section 3.

5. Cell-Centered Schemes. In many fluid mechanics calculations, it is convenient to approximate some physical variables at cell-centers rather than at nodes or vertices of the mesh. In one dimension, a cell-centered mesh is constructed by placing the nodes $x_{1}\left(>x_{0}\right)$ and $x_{N-1}\left(<x_{N}\right)$ on the interval boundaries. Quantities of interest are then approximated at $x_{i-1 / 2}=\frac{1}{2}\left(x_{i}+x_{i-1}\right), i=1, \ldots, N$. Actually, we have been dealing with cell-centered quantities all along; it would just have been confusing to point them out as such. For example, all the truncation errors in the discussion of first-order systems in Section 2 are cell-centered. In this section, $V$ will still denote a vector, but the components may be given as $(V)_{i} \equiv v_{i}$, as before, if it is a vertex-centered quantity, or $(V)_{i-1 / 2} \equiv v_{i-1 / 2}$ if it is a cell-centered quantity. In each case, the subscript denotes the variable's association with the mesh point $x_{i}$ or the cell-centered point $x_{i-1 / 2}$. The cell-centered mesh distances will be written as $\Delta_{i-1 / 2} \equiv \frac{1}{2}\left(\Delta_{i}+\Delta_{i-1}\right)=\left(x_{i-1 / 2}-x_{i-3 / 2}\right)$.

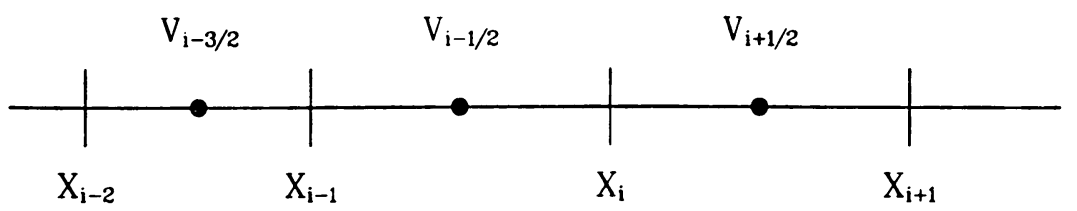

FIGURE 5.1

Portion of a cell-centered mesh.

We will examine the solution of the linear, second-order boundary value problem (1.1) and (1.2a,b). For cell-centered schemes, the usual approximation to $y^{\prime \prime}$ can be explained using Figure 5.1. Assume that the left difference $\left[\left(v_{i-1 / 2}-v_{i-3 / 2}\right) / \Delta_{i-1 / 2}\right]$ is an accurate representation of $y^{\prime}$ at $x_{i-1}$ and, similarly, $\left[\left(v_{i+1 / 2}-v_{i-1 / 2}\right) / \Delta_{i+1 / 2}\right]$ at $x_{i}$. Now, the difference of these first differences gives us an approximation to $y^{\prime \prime}$ at $x_{i-1 / 2}$ :

$$
\frac{1}{\Delta_{i}}\left[\frac{v_{i+1 / 2}-v_{i-1 / 2}}{\Delta_{i+1 / 2}}-\frac{v_{i-1 / 2}-v_{i-3 / 2}}{\Delta_{i-1 / 2}}\right] \equiv\left(D_{2}^{c c} V\right)_{i-1 / 2},
$$

where superscript $c c$ denotes cell-centered. Many people have noted (see [23]) that (5.1) is, in general, an inconsistent approximation to the second derivative on a nonuniform mesh. That is,

$$
\begin{aligned}
\left(D_{2}^{c c} Y\right)_{i-1 / 2}= & y^{\prime \prime}\left(x_{i-1 / 2}\right)+\frac{\Delta_{i+1}-2 \Delta_{i}+\Delta_{i-1}}{4 \Delta_{i}} y^{\prime \prime}\left(x_{i-1 / 2}\right) \\
& +\frac{\Delta_{i+1}^{2}+2 \Delta_{i+1} \Delta_{i}-2 \Delta_{i} \Delta_{i-1}-\Delta_{i-1}^{2}}{24 \Delta_{i}} y^{\prime \prime \prime}\left(x_{i-1 / 2}\right)+\cdots,
\end{aligned}
$$

and the approximation is inconsistent. We will show that some difference schemes still yield $O\left(\Delta^{2}\right)$ solution errors in spite of this truncation error. 
We will employ first-order systems, in an unorthodox way, to help us find the correct way to analyze these methods. The major complications will arise from the $O$ (1) truncation error term in (5.2). Of itself, it is easily treated, but its effect on boundary condition approximations and on difference approximations to $a y^{\prime}+b y$ will be considerable.

First, we notice that (just as for the vertex-centered scheme) the cell-centered approximation to $y^{\prime \prime}$ is given by multiplying the matrices $D_{0}$ and $D_{1}$ together but in the reverse order. That is,

$$
\left(D_{2}^{c c} V\right)_{i-1 / 2} \equiv\left(D_{0} D_{1} V\right)_{i-1 / 2}, \quad i=2, \ldots, N-1,
$$

where $D_{0}$ and $D_{1}$ are precisely as defined in (2.4a) and (2.11a), except that the first and last rows of $D_{0}$ are deleted.

Consider the solution of the constant-coefficient equation,

$$
y^{\prime \prime}+a y^{\prime}+b y=f \text {. }
$$

Written as a first-order system, we can approximate this equation by

$$
\begin{gathered}
D_{1} V=\overline{A_{1}} W \\
D_{1} W=-a \overline{A_{1}} W-b \overline{A_{1}} V+F
\end{gathered}
$$

where $(F)_{i}=f_{i}$ and

$$
\overline{A_{1}} \equiv\left[\begin{array}{ccc}
\frac{\Delta_{2}}{\Delta_{1}+\Delta_{2}} & \frac{\Delta_{1}}{\Delta_{1}+\Delta_{2}} & \\
& \frac{\Delta_{3}}{\Delta_{2}+\Delta_{3}} & \frac{\Delta_{2}}{\Delta_{2}+\Delta_{3}} \\
& & \ddots
\end{array}\right] .
$$

Replacing $V$ and $W$ with $Y$ and $Y^{\prime}$ (exact derivative), we get

$$
\begin{gathered}
D_{1} Y=\overline{A_{1}} Y^{\prime}+D_{1} T_{1}+\overline{T_{2}}, \\
D_{1} Y^{\prime}=-a \overline{A_{1}} Y^{\prime}-b \overline{A_{1}} Y+F+D_{1} \bar{T}_{3}+\bar{T}_{4},
\end{gathered}
$$

where

$$
\begin{gathered}
\left(T_{1}\right)_{i-1 / 2}=\frac{1}{8} \Delta_{i}^{2} y_{i-1 / 2}^{\prime \prime} \\
\left(\bar{T}_{2}\right)_{i}=-\frac{1}{24}\left(2 \Delta_{i+1}^{2}+\Delta_{i+1} \Delta_{i}+2 \Delta_{i}^{2}\right) y_{i}^{\prime \prime \prime}+O\left(\Delta^{3}\right) \\
\left(\bar{T}_{3}\right)_{i-1 / 2}=\frac{1}{8} \Delta_{i}^{2} y_{i-1 / 2}^{\prime \prime \prime} \\
\left(\bar{T}_{4}\right)_{i}=\frac{1}{24}\left(2 \Delta_{i+1}^{2}+\Delta_{i+1} \Delta_{i}+2 \Delta_{i}^{2}\right)\left(a y_{i}^{\prime \prime \prime}+b y_{i}^{\prime \prime}\right)+O\left(\Delta^{3}\right)
\end{gathered}
$$

We can find appropriate commuting pairs of matrices,

$$
A_{0} D_{1}=D_{0} \overline{A_{1}}
$$

which allow us to combine $(5.6 \mathrm{a}, \mathrm{b})$ into

$$
\begin{aligned}
& D_{0} D_{1} Y+a D_{0} \overline{A_{1}} Y+b A_{0} \overline{A_{1}} Y \\
& \quad=A_{0} F+D_{0} D_{1} T_{1}+D_{0}\left(\bar{T}_{2}+\overline{A_{1}} \bar{T}_{3}+a \overline{A_{1}} T_{1}\right)+A_{0}\left(\bar{T}_{4}+a \bar{T}_{2}\right) .
\end{aligned}
$$

Note that (5.9) defines a three-point, cell-centered difference scheme approximating this constant-coefficient differential equation. 
Now we will examine the pointwise error equation implied by (5.9), namely,

$$
\begin{aligned}
& D_{0} D_{1} E+a D_{0} \overline{A_{1}} E+b A_{0} \overline{A_{1}} E \\
& \quad=D_{0} D_{1} T_{1}+D_{0}\left(\bar{T}_{2}+\overline{A_{1}} \bar{T}_{3}+a \overline{A_{1}} T_{1}\right)+A_{0}\left(\bar{T}_{4}+a \bar{T}_{2}\right),
\end{aligned}
$$

in order to see how the analysis should proceed in a more general case. In the course of this examination, we will point out the purpose of each of the four lemmas in Appendix A leading to the main result of this section, Theorem 5.1. (For Appendix A, see the supplements section at the end of this issue.) The first question to consider is what sort of truncation error can be put in a form similar to (5.9). This question will be answered in Lemma A.1.

The leading term in the truncation error is easily removed by defining

Substituting this into (5.10), we have

$$
E=T_{1}+E_{1} \text {. }
$$

$$
\begin{aligned}
& D_{0} D_{1} E_{1}+a D_{0} \overline{A_{1}} E_{1}+b A_{0} \overline{A_{1}} E_{1} \\
& \quad=D_{0}\left(\bar{T}_{2}+\overline{A_{1}} \overline{T_{3}}+a \overline{A_{1}} T_{1}\right)+A_{0}\left(\bar{T}_{4}+a \bar{T}_{2}\right)-\left(a D_{0} \overline{A_{1}}+b A_{0} \overline{A_{1}}\right) T_{1} .
\end{aligned}
$$

We recognize the last term on the right-hand side of this equation to be $-L_{1} T_{1}$, where $L_{1}$ is a difference approximation to $a y^{\prime}+b y$. Rewriting this equation gives us

$$
\left(D_{0} D_{1}+L_{1}\right) E_{1}=D_{0}\left(\bar{T}_{2}+\bar{A}_{1} \bar{T}_{3}+a \bar{A}_{1} T_{1}\right)+A_{0}\left(\bar{T}_{4}+a \bar{T}_{2}\right)-L_{1} T_{1} .
$$

Note that this error equation has the same form as those encountered in Sections 2 and 3 if we can rewrite $L_{1} T_{1}$ as $D_{0} \bar{T}_{5}+\bar{T}_{6}$, where $\bar{T}_{5}$ and $\bar{T}_{6}$ are $O\left(\Delta^{2}\right)$. Lemma A.2 will derive conditions sufficient for (5.11) to be written as

$$
\left(D_{0} D_{1}+L_{1}\right) E_{1}=D_{0} T_{2}+A_{0} T_{4}+\bar{T}_{6},
$$

where, in our particular case, $T_{2}=\bar{T}_{2}+\bar{A}_{1} \bar{T}_{3}+a \bar{A}_{1} T_{1}+\bar{T}_{5}, T_{4}=\bar{T}_{4}+a \bar{T}_{2}$, and $\bar{T}_{6}$ are $O\left(\Delta^{2}\right)$.

The remainder of the proof proceeds exactly like that in Section 3. Let

$$
E_{1}=E_{2}+E_{3}
$$

$$
D_{1} E_{2}=T_{2} \text {. }
$$

Lemma A.3 will prove that $E_{2}=O\left(\Delta^{2}\right)$. Substituting (5.13a) into (5.12) yields the following equation for $E_{3}$,

$$
\left(D_{0} D_{1}+L_{1}\right) E_{3}=A_{0} \bar{T}_{4}+\bar{T}_{6}-L_{1} E_{2} .
$$

Finally, Lemma A.4 will show that under mild assumptions on $L_{1}$, we have $L_{1} E_{2}=O\left(\Delta^{2}\right)$, and thus stability (plus boundary condition stuff) will yield secondorder accuracy for these schemes. These four lemmas are proven in Appendix A, but in some cases are tedious and ugly and should be passed over except by the most resolute readers.

The main result of this section utilizes the results in Lemmas A.2-A.4. This theorem corresponds to Corollary 3.3 for vertex-centered meshes. The discrete boundary conditions will be written as

$$
B_{0} V=\alpha_{1} v_{1 / 2}+\beta_{1} v_{3 / 2}+\gamma_{1} v_{5 / 2},
$$

$$
B_{1} V=\alpha_{N} v_{N-5 / 2}+\beta_{N} v_{N-3 / 2}+\gamma_{N} v_{N-1 / 2}
$$


The discrete approximation to the differential equation is given by

$$
\left(\left[D_{0} D_{1}+L_{1}\right] V\right)_{i-1 / 2}=(F)_{i-1 / 2}, \quad i=2, \ldots, N-1,
$$

where $(F)_{i-1 / 2}$ may stand for a linear combination of the function $f(x)$ evaluated at certain points.

THEOREM 5.1. Let the difference scheme (5.14a,b) and (5.15) satisfy the hypotheses of Lemmas A.2 and A.4 and be stable for all meshes in some class M. Further, let the difference scheme have the following properties:

(i) the truncation error for the differential equation approximation (5.15) has the form

$$
\left(D_{0} D_{1}+L_{1}\right) Y=F+D_{0} D_{1} T_{1}+D_{0} \bar{T}_{2}+\bar{T}_{3}
$$

where $T_{1} \bar{T}_{2} \bar{T}_{3}$ are all $O\left(\Delta^{2}\right)$;

(ii) the truncation error for the boundary condition approximations has the form,

$$
\begin{gathered}
B_{0}\left(Y-T_{1}\right)=b_{02}+\left(\bar{T}_{3}\right)_{1 / 2}, \\
B_{1}\left(Y-T_{1}\right)=b_{12}+\left(\bar{T}_{3}\right)_{N-1 / 2},
\end{gathered}
$$

where $\left(\bar{T}_{3}\right)_{1 / 2}$ and $\left(\bar{T}_{3}\right)_{N-1 / 2}$ are $O\left(\Delta^{2}\right)$; and

(iii) the discrete boundary conditions satisfy

$$
\begin{gathered}
\left|\alpha_{1}+\beta_{1}+\gamma_{1}\right|,\left|\Delta_{1 / 2} \alpha_{1}\right|,\left|\Delta_{3 / 2} \gamma_{1}\right| \leqslant C, \\
\left|\alpha_{N}+\beta_{N}+\gamma_{N}\right|,\left|\Delta_{N-3 / 2} \alpha_{N}\right|,\left|\Delta_{N-1 / 2} \gamma_{N}\right| \leqslant C .
\end{gathered}
$$

Then,

$$
\|Y-V\|_{\infty} \leqslant C \Delta_{\max }^{2}
$$

for all meshes in $M$.

Proof. From (5.16), we can derive the pointwise error equation for the differential equation,

$$
\left(D_{0} D_{1}+L_{1}\right) E=D_{0} D_{1} T_{1}+D_{0} \bar{T}_{2}+\bar{T}_{3} \text {. }
$$

Let $E=T_{1}+E_{1}$, and we get

$$
\left(D_{0} D_{1}+L_{1}\right) E_{1}=D_{0} \bar{T}_{2}+\bar{T}_{3}-L_{1} T_{1} .
$$

However, Lemma A.2 allows us to write

$$
L_{1} T_{1}=D_{0} \bar{T}_{1}-\bar{T}_{6}
$$

where $\bar{T}_{1}, \bar{T}_{6}=O\left(\Delta^{2}\right)$. Defining $\bar{T}_{2}-\bar{T}_{1}=T_{2}$, we have

$$
\left(D_{0} D_{1}+L_{1}\right) E_{1}=D_{0} T_{2}+\left(\bar{T}_{3}+\bar{T}_{6}\right) \text {. }
$$

Let $E_{1}=E_{2}+E_{3}$, where $E_{2}$ is defined in Lemma A.3; then

$$
\left(D_{0} D_{1}+L_{1}\right) E_{3}=\bar{T}_{3}+\bar{T}_{6}-L_{1} E_{2} .
$$

Finally, Lemma A.4 shows that $L_{1} E_{2}=O\left(\Delta^{2}\right)$; thus we can write

$$
\left(\left[D_{0} D_{1}+L_{1}\right] E_{3}\right)_{i-1 / 2}=\left(T_{3}\right)_{i-1 / 2}, \quad i=2, \ldots, N-1,
$$

where $\left(T_{3}\right)_{i-1 / 2}=\left(\bar{T}_{3}+\bar{T}_{6}-L_{1} E_{2}\right)_{i-1 / 2}$. 
The pointwise error equations for the boundary conditions are

$$
B_{0} E=B_{0} T_{1}+\left(\bar{T}_{3}\right)_{1 / 2}, \quad B_{1} E=B_{1} T_{1}+\left(\bar{T}_{3}\right)_{N-1 / 2} .
$$

Substituting $E=T_{1}+E_{2}+E_{3}$ (unraveling the substitutions made for the discrete differential equations), we have

$$
B_{0} E_{3}=\left(\bar{T}_{3}\right)_{1 / 2}-B_{0} E_{2}, \quad B_{1} E_{3}=\left(\bar{T}_{3}\right)_{N-1 / 2}-B_{1} E_{2} .
$$

Property (iii) in the hypothesis is sufficient (see the proof of Lemma A.4) to show that

$$
\left|B_{0} E_{2}\right|,\left|B_{1} E_{2}\right| \leqslant C \Delta_{\max }^{2}
$$

Thus, we define

$$
\left(T_{3}\right)_{1 / 2}=\left(\bar{T}_{3}\right)_{1 / 2}-B_{0} E_{2}, \quad\left(T_{3}\right)_{N-1 / 2}=\left(\bar{T}_{3}\right)_{N-1 / 2}-B_{1} E_{2},
$$

and we know that

$$
\left\|T_{3}\right\|_{\infty} \leqslant C \Delta_{\max }^{2}
$$

Pulling all this together, we have

$$
\begin{gathered}
B_{0} E_{3}=\left(T_{3}\right)_{1 / 2}, \\
\left(\left[D_{0} D_{1}+L_{1}\right] E_{3}\right)_{i-1 / 2}=\left(T_{3}\right)_{i-1 / 2}, \quad i=2, \ldots, N-1, \\
B_{1} E_{3}=\left(T_{3}\right)_{N-1 / 2},
\end{gathered}
$$

and stability of the difference scheme yields

$$
\left\|E_{3}\right\| \leqslant C \Delta_{\max }^{2} .
$$

The error is given by

$$
E=Y-V=T_{1}+E_{2}+E_{3},
$$

where we already know that $T_{1}, E_{2}$ are $O\left(\Delta^{2}\right)$, so

$$
\|Y-V\|_{\infty} \leqslant C \Delta_{\max }^{2}
$$

This theorem points out that the boundary conditions for cell-centered schemes must be handled carefully in order to preserve second-order accuracy in the solution. For purely Dirichlet boundary conditions $\left(b_{01}=b_{11}=0\right)$, the condition that

$$
B_{i}\left(Y-T_{1}\right)-b_{i 2}=O\left(\Delta^{2}\right)
$$

will not alter the 'usual' approximation because $B_{i} T_{1}=O\left(\Delta^{2}\right)$. However, for mixed boundary conditions $\left(b_{01} \neq 0\right.$ or $b_{11} \neq 0$ ), failure to satisfy this condition will result in a solution that is first-order accurate. As an example, we will derive the correct boundary conditions at $x=0$ for the difference scheme (5.4a,b). Recall from (5.7a) that $\left(T_{1}\right)_{i-1 / 2}=\frac{1}{8} \Delta_{i}^{2} y_{i-1 / 2}^{\prime \prime}$. Thus, we have

$\alpha_{1}\left(y_{1 / 2}-\frac{1}{8} \Delta_{1}^{2} y_{1 / 2}^{\prime \prime}\right)+\beta_{1}\left(y_{3 / 2}-\frac{1}{8} \Delta_{2}^{2} y_{3 / 2}^{\prime \prime}\right)+\gamma_{1}\left(y_{5 / 2}-\frac{1}{8} \Delta_{3}^{2} y_{5 / 2}^{\prime \prime}\right)-b_{02}=O\left(\Delta^{2}\right)$.

Expanding in Taylor series about $x=0$ gives us

$$
\begin{aligned}
\left(\alpha_{1}+\beta_{1}+\gamma_{1}\right) y_{1}+\left(-\frac{1}{2} \Delta_{1} \alpha_{1}+\right. & \left.\frac{1}{2} \Delta_{2} \beta_{1}+\left[\frac{1}{2} \Delta_{2}+\Delta_{3 / 2}\right] \gamma_{1}\right) y_{1}^{\prime} \\
& +\left(\Delta_{2} \Delta_{5 / 2}\right) \gamma_{1} y_{1}^{\prime \prime}=b_{00} y_{1}+b_{01} y_{1}^{\prime} O\left(\Delta^{2}\right) .
\end{aligned}
$$


A discrete boundary condition satisfying this equation is

$$
b_{00}\left[\frac{\Delta_{1} v_{3 / 2}+\Delta_{2} v_{1 / 2}}{\Delta_{2}+\Delta_{1}}\right]+b_{01}\left[\frac{v_{3 / 2}-v_{1 / 2}}{\Delta_{3 / 2}}\right]=b_{02} \text {. }
$$

At $x=1$, we have a corresponding condition

(5.20b) $b_{10}\left[\frac{\Delta_{N-1} v_{N-1 / 2}+\Delta_{N} v_{N-3 / 2}}{\Delta_{N}+\Delta_{N-1}}\right]+b_{11}\left[\frac{v_{N-1 / 2}-v_{N-3 / 2}}{\Delta_{N-1 / 2}}\right]=b_{12}$.

Numerical evidence leads us to believe that if property (iii) in Theorem 5.1 is violated, then the solution will not be second-order accurate. That is, this is a genuine constraint and not an artifact of the proof.

6. Summary. We have shown that most reasonable, vertex-centered difference schemes for linear and quasilinear boundary value problems yield second-order accurate solutions on nonuniform meshes. This result is derived in spite of the fact that the truncation error for these compact schemes is only first-order. Each of these results can be extended to systems of second-order equations without substantial difficulty.

The numerical results shown in Figures 6.1-6.4, following, are solutions to the boundary value problem

$$
(3 x+1)^{2} y^{\prime \prime}+9 y^{\prime}+9\left[\frac{1}{4}(3 x+1)^{2}-1\right] y=0
$$

$$
y^{\prime}(0), y(1) \text { given. }
$$

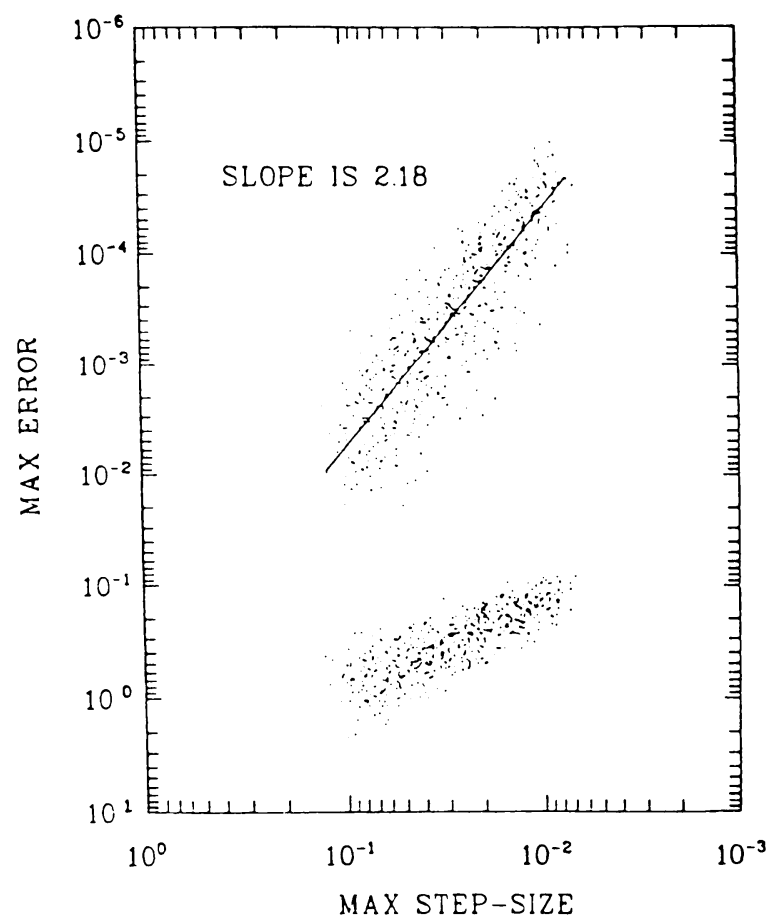

FIGURE 6.1

First-order system. 


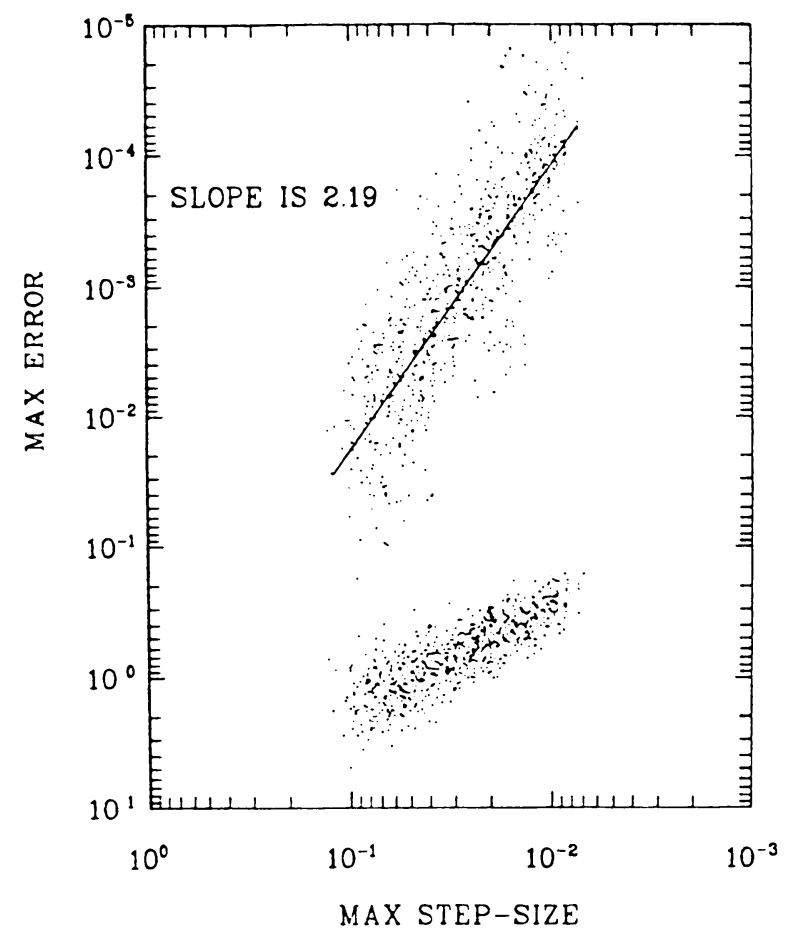

Figure 6.2

Pearson's scheme.

In each figure, the differential equation is approximated on 700 random meshes ( $N-1$ points placed in $(0,1)$ at random) and the maximum error is plotted versus the maximum step size; $N$ ranges from 100 to 800 .

Figure 6.1 is the vertex-centered difference scheme derived from a first-order system (see (2.14)). The actual solution error for each mesh appears in the upper portion of this plot; the straight line "through" these points is the least-squares fit to the solution error. In the lower portion of this plot is the truncation error for each mesh. The slope of the least-squares fit to these points is 0.78 . Figure 6.2 is the vertex-centered difference scheme used by Pearson [21] and displays similar information as Figure 6.1.

In both these figures, the results of Theorem 3.2 and Corollary 3.3 are borne out. That is, the truncation error is of lower order than the solution error. For these meshes, the local mesh ratio $\left(\Delta_{\max } / \Delta_{\min }\right)$ may be as large as $10^{7}$, so this is a very stringent test.

We have also shown that some cell-centered schemes for linear boundary value problems (quasilinear equations should pose no essential difficulty) yield secondorder accurate solutions. This is in spite of the fact that these schemes are inconsistent. Most important, this result reveals that mixed boundary conditions must be approximated carefully in order to preserve this accuracy.

Figures 6.3 and 6.4 show the solution error in solving (6.1) by the cell-centered scheme (5.1) and (A.7). In Figure 6.3, the boundary conditions are approximated correctly using $(5.20 \mathrm{a}, \mathrm{b})$, and the solution error is second order. In Figure 6.4, the 


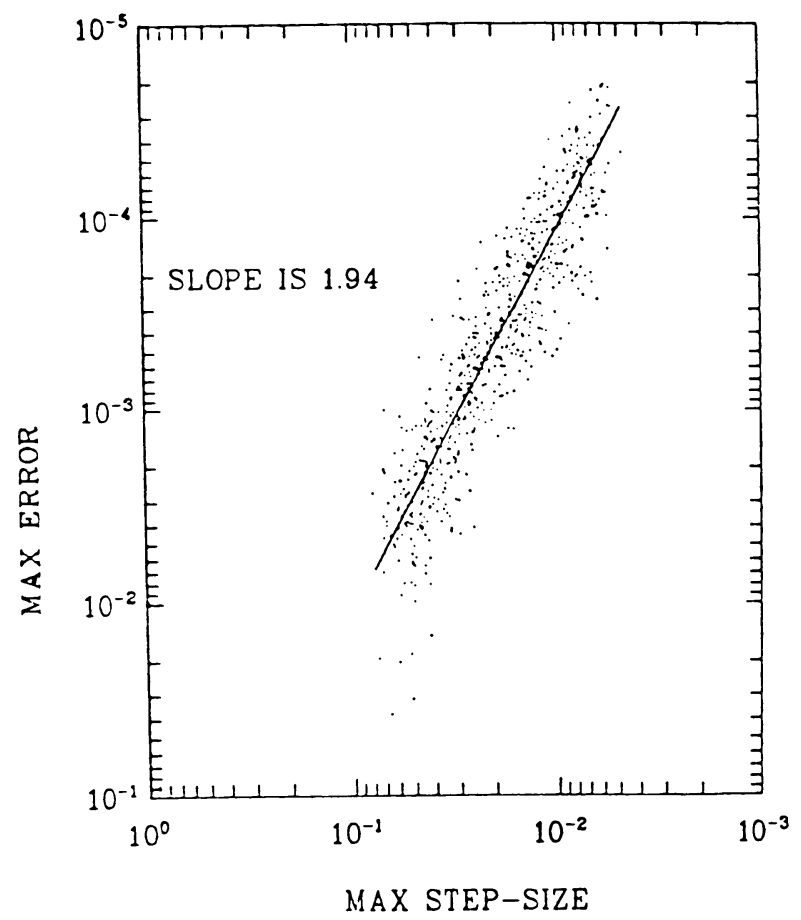

FIGURE 6.3

Correct cell-centered boundary conditions.

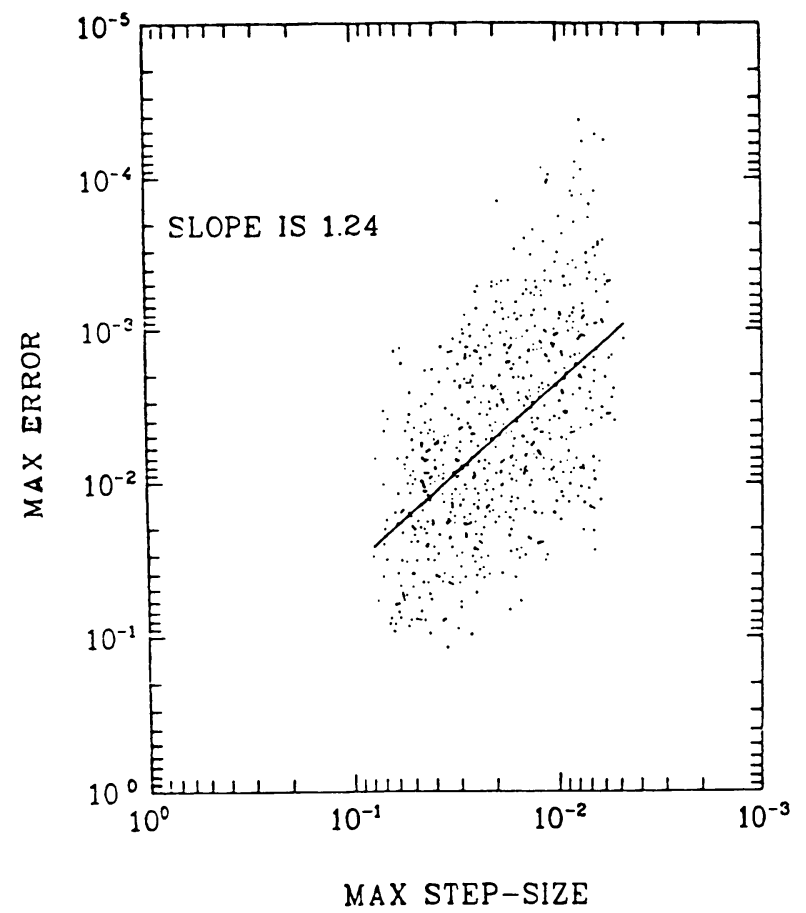

FIGURE 6.4

Second-order cell-centered boundary conditions. 
boundary conditions are approximated correct to second order:

$$
\begin{aligned}
& B_{0} Y=b_{00} y(0)+b_{01} y^{\prime}(0)+O\left(\Delta^{2}\right), \\
& B_{1} Y=b_{10} y(1)+b_{11} y^{\prime}(1)+O\left(\Delta^{2}\right),
\end{aligned}
$$

but they violate property (ii) of Theorem 5.1. Here, the solution error has degraded to first order, although it still remains of higher order than the truncation error.

Motivated by the elimination of auxiliary unknowns in first-order systems, we have developed mathematical tools that, together with stability arguments, yield better estimates of accuracy. Our work has shown the limitations of the standard stability-consistency arguments when applied to nonuniform meshes. On the other hand, these results can also be obtained by combining stability with summation by parts; see, e.g., Kreiss et al. [16]. In addition, one should note the alternative approach of Grigorieff [9] utilizing stability and consistency with respect to a Spijker norm.

The reduction process described in Section 2 provides an efficient computational algorithm (see Manteuffel and White [19]). In addition, the concept of pairwise commuting matrices can be used to extend this analysis to higher-order differential equations. These results will appear in a subsequent paper.

Finally, it is easy to see how to extend these results to separable difference schemes on separable meshes. For example, consider the solution of the heat equation

$$
u_{t}=u_{x x}
$$

on the grid $\left(x_{i}, t_{k}\right)$. A Crank-Nicolson scheme for approximating (6.3) is

$$
\left(L_{h} V\right)_{i}^{k} \equiv \frac{v_{i}^{k}-v_{i}^{k-1}}{\Delta t_{k}}-\frac{1}{2}\left(D_{2} V^{k}+D_{2} V^{k-1}\right)_{i} \equiv 0,
$$

where $D_{2}$ is the second divided difference (1.3a), and

$$
V^{k}=\left(v_{0}^{k} v_{1}^{k} \cdots v_{N}^{k}\right)^{T} \text {. }
$$

In order to show that the error is second order, we follow the prescription given in Section 3.

Let

$$
\left(E_{1}^{k}\right)_{i}=\frac{1}{3} \sum_{j=1}^{i}\left(\Delta_{j}\right)^{3} u_{x x x}\left(x_{j-1 / 2}, t^{k}\right),
$$

and $E^{k}=E_{1}^{k}+E_{2}^{k}$. The resulting equation for $E_{2}$ is

$$
\left(L_{h} E_{2}\right)_{i}^{k}=\left(T_{2}\right)_{i}^{k}-\frac{\left(E_{1}^{k}\right)_{i}-\left(E_{1}^{k-1}\right)_{i}}{\Delta t_{k}},
$$

where $T_{2}=O\left(\Delta_{\max }^{2}+\Delta t_{\max }^{2}\right)$. Clearly, if the time-difference operator applied to $E_{1}$ is $O\left(\Delta^{2}\right)$, then stability will yield the desired result. Substituting from (6.4) yields

$$
\begin{aligned}
\frac{\left(E_{1}^{k}\right)_{i}-\left(E_{1}^{k-1}\right)_{i}}{\Delta t_{k}} & =\frac{1}{3} \sum_{j=1}^{i}\left(\Delta_{j}\right)^{3}\left[\frac{u_{x x x}\left(x_{j-1 / 2}, t_{k}\right)-u_{x x x}\left(x_{j-1 / 2}, t_{k-1}\right)}{\Delta t_{k}}\right] \\
& \approx \frac{1}{3} \sum_{j=1}^{i}\left(\Delta_{j}\right)^{3} u_{x x x t}\left(x_{j-1 / 2}, t_{k}\right) .
\end{aligned}
$$


Thus, if the exact solution is sufficiently smooth, then

$$
\left|\frac{\left(E_{1}^{k}\right)_{i}-\left(E_{1}^{k-1}\right)_{i}}{\Delta t_{k}}\right| \leqslant C \Delta_{\text {max }}^{2} .
$$

This same argument will work for difference schemes approximating elliptic, hyperbolic, or parabolic equations provided (1) that the solution is smooth, and (2) that the difference operators in one direction are not affected by the nonuniform mesh in another direction.

Computing and Communications Division

Los Alamos National Laboratory

Los Alamos, New Mexico 87545

1. C. M. Ablow \& S. SChechter, “Campylotropic coordinates," J. Comput. Phys., v. 27, 1978, pp. $351-362$.

2. T. H. СноNG, "A variable mesh finite difference method for solving a class of parabolic differential equations in one space variable," SIAM J. Numer. Anal., v. 15, 1978, pp. 835-857.

3. Melvyn Ciment, Stephen W. Leventhal \& Bernard C. Weinberg, "The operator compact implicit method for parabolic equations," J. Comput. Phys., v. 28, 1978, pp. 135-166.

4. STEPHEN F. Davis \& JosePh E. FlaherTy, "An adaptive finite element method for initial-boundary value problems for partial differential equations," SIAM J. Sci. Statist. Comput., v. 3, 1982, pp. 6-27.

5. V. E. DENNY \& R. B. LANDIS, "A new method for solving two-point boundary value problems using optimal node distribution," J. Comput. Phys., v. 9, 1972, pp. 120-137.

6. EUGENIA KaUNAX DE Rivas, "On the use of nonuniform grids in finite difference equations," $J$. Comput. Phys., v. 10, 1972, pp. 202-210.

7. Eusebius J. DOEDEL, "The construction of finite difference approximations to ordinary differential equations," SIAM J. Numer. Anal., v. 15, 1978, pp. 450-465.

8. EusEBIUS J. DOEDEL, "Finite difference methods for nonlinear two-point boundary value problems," SIAM J. Numer. Anal., v. 16, 1979, pp. 173-185.

9. ROLF D. GRIGORIEFF, "Some stability inequalities for compact finite difference schemes," typescript.

10. J. D. Hoffman, "Relationship between the truncation errors of centered finite-difference approximations on uniform and nonuniform meshes," J. Comput. Phys., v. 46, 1982, pp. 469-474.

11. Eugene IsaACson \& H. B. Keller, Analysis of Numerical Methods, Wiley, New York, 1966.

12. HeRbert B. Keller, "Accurate difference methods for linear ordinary differential systems subject to linear constraints," SIAM J. Numer. Anal., v. 6, 1969, pp. 8-30.

13. H. B. Keller \& A. B. White, JR., "Difference methods for boundary value problems in ordinary differential equations," SIAM J. Numer. Anal., v. 12, 1975, pp. 791-802.

14. Herbert B. Keller, Numerical Solution of Two Point Boundary Value Problems, SIAM, Regional Conference Series in Applied Mathematics, 24.

15. H.-O. KreISS, "Difference approximations for boundary and eigenvalue problems for ordinary differential equations," Math. Comp., v. 26, 1972, pp. 605-624.

16. H.-O. Kreiss, T. A. Manteuffel, B. Swartz, B. Wendroff \& A. B. White, JR., "Supra-convergent schemes on irregular grids," Math. Comp., v. 47, 1986, pp. 537-554.

17. DAVID LEVERMORE, private communication.

18. R. E. LYNCH \& J. R. RICE, The HODIE method, Report C5D-TR 188, Dept. of Computer Science, Purdue University, W. Lafayette, Indiana.

19. T. A. Manteuffel \& A. B. White, JR., "On the efficient solution of systems of second-order boundary value problems," SIAM J. Numer. Anal. (To appear.)

20. M. R. OSBORNE, "Minimizing truncation error in finite difference approximations to ordinary differential equations," Math. Comp., v. 21, 1967, pp. 133-145.

21. Carl E. Pearson, "On a differential equation of boundary layer type," J. Math. Phys., v. 47, 1968, pp. 134-154.

22. Blair K. Swartz, "The construction and comparison of finite difference analogs of some finite element schemes," in Mathematical Aspects of Finite Elements Partial Differential Equation (1974).

23. A. N. Tiknonov \& A. A. Samarskil, "Homogeneous difference schemes on nonuniform nets," $Z h$. Vychisl. Mat. i Mat. Fiz., v. 1, 1962, English translation (Russian).

24. A. B. White, JR., "On selection of equidistributing meshes for two-point boundary value problems," SIAM J. Numer. Anal., v. 16, 1979, pp. 472-502. 


\section{Supplement to \\ The Numerical Solution of Second-Order Boundary Value Problems on Nonuniform Meshes}

By Thomas A. Manteuffel and Andrew B. White, Jr.

\section{APPENDIX A}

LEMMA A.1. Let the leading terms in the truncation error be

(A.1) $\quad(T)_{i-1 / 2}=\frac{\Delta_{i+1}-2 \Delta_{i}+\Delta_{i-1}}{\Delta} p\left(x_{i-1 / 2}\right)$

$$
+\frac{F\left(\Delta_{i+1}, \Delta_{1}\right)-F\left(\Delta_{i}, \Delta_{i-1}\right)}{\Delta_{i}} q\left(x_{i-1 / 2}\right)+O\left(\Delta^{2}\right), i=2, \ldots . N-1 .
$$

where $p(x) \varepsilon C^{2}(0,1)$ and $q(x) \in C^{1}(0,1)$ and

$$
\max _{1 \leq \leq N-1}\left|F\left(\Delta_{i+1} . \Delta_{i}\right)\right| \leq C \Delta_{\max }^{2}
$$

for some class of meshes $M$. Then we can write

(A.2)

$$
T=D_{0} D_{1} T_{1}+D_{0} T_{2}+T_{3},
$$

where $\|T\|, C \Delta_{\text {max }}^{2}, i=1,2,3$, for all meshes in $M$

Proof. First, we will break the proof into two parts: let

$$
T=\Sigma_{1}+\Sigma_{2} \text {. }
$$

where

$$
\left(\Sigma_{1}\right)_{i-1 / 2}=\frac{\Delta_{i+1}-2 \Delta_{i}+\Delta_{i-1}}{\Delta_{i}} p\left(x_{i-1 / 2}\right)
$$

and

$$
\left(\Sigma_{2}\right)_{i-1 / 2}=\frac{F\left(\Delta_{i+1}, \Delta_{i}\right)-F\left(\Delta_{i}, \Delta_{i-1}\right)}{\Delta_{i}} q\left(x_{i-1 / 2}\right)
$$

Using Taylor series expansion on $p_{1-1 / 2}$ gives us

$$
\begin{aligned}
\left(\Sigma_{1}\right)_{i-1 / 2} & =\frac{\Delta_{i+1}-\Delta_{i}}{\Delta_{i}} p_{i}-\frac{\Delta_{i}-\Delta_{i-1}}{\Delta_{i}} p_{i-1} \\
& -\frac{1}{2}\left(\Delta_{i+1}-\Delta_{i}\right) p_{i}^{\prime}-\frac{1}{2}\left(\Delta_{i}-\Delta_{i-1}\right) p_{i-1}^{\prime} \\
& +\frac{1}{8}\left(\Delta_{i+1}-\Delta_{i}\right) \Delta_{i} p^{\prime \prime}-\frac{1}{8}\left(\Delta_{i}-\Delta_{i-1}\right) \Delta_{i} p^{\prime \prime} .
\end{aligned}
$$

(A.3)

where throughout this proof we will denote intermediate values by leaving the function $p$ or $q$ without subscripts. Again using Taylor series, for the first line of (A.3) we have

$$
\frac{\Delta_{i+1}-\Delta_{i}}{\Delta_{i}} p_{i}-\frac{\Delta_{i}-\Delta_{i-1}}{\Delta_{i}} p_{i-1}=\left(-\frac{1}{\Delta_{i}} \frac{1}{\Delta_{i}}\right)\left(\begin{array}{l}
\frac{\Delta_{i}^{2} p_{i-12}-\Delta_{i-1}^{2} p_{i-32}}{\Delta_{i}+\Delta_{i-1}} \\
\frac{\Delta_{i+1}^{2} p_{i+1 / 2}-\Delta_{i}^{2} p_{i-12}}{\Delta_{i+1}+\Delta_{i}}
\end{array}\right)
$$

(A.4)

$$
+\frac{1}{2}\left(-\frac{1}{\Delta_{i}} \frac{1}{\Delta_{i}}\right)\left(\begin{array}{l}
-\frac{\Delta_{i}^{3}}{\Delta_{i}+\Delta_{i-1}} p^{\prime}-\frac{\Delta_{i-1}^{3}}{\Delta_{i}+\Delta_{i-1}} p^{\prime} \\
-\frac{\Delta_{i+1}^{3}}{\Delta_{i+1}+\Delta_{i}} p^{\prime}-\frac{\Delta_{i}^{3}}{\Delta_{i+1}+\Delta_{i}} p^{\prime}
\end{array}\right)
$$



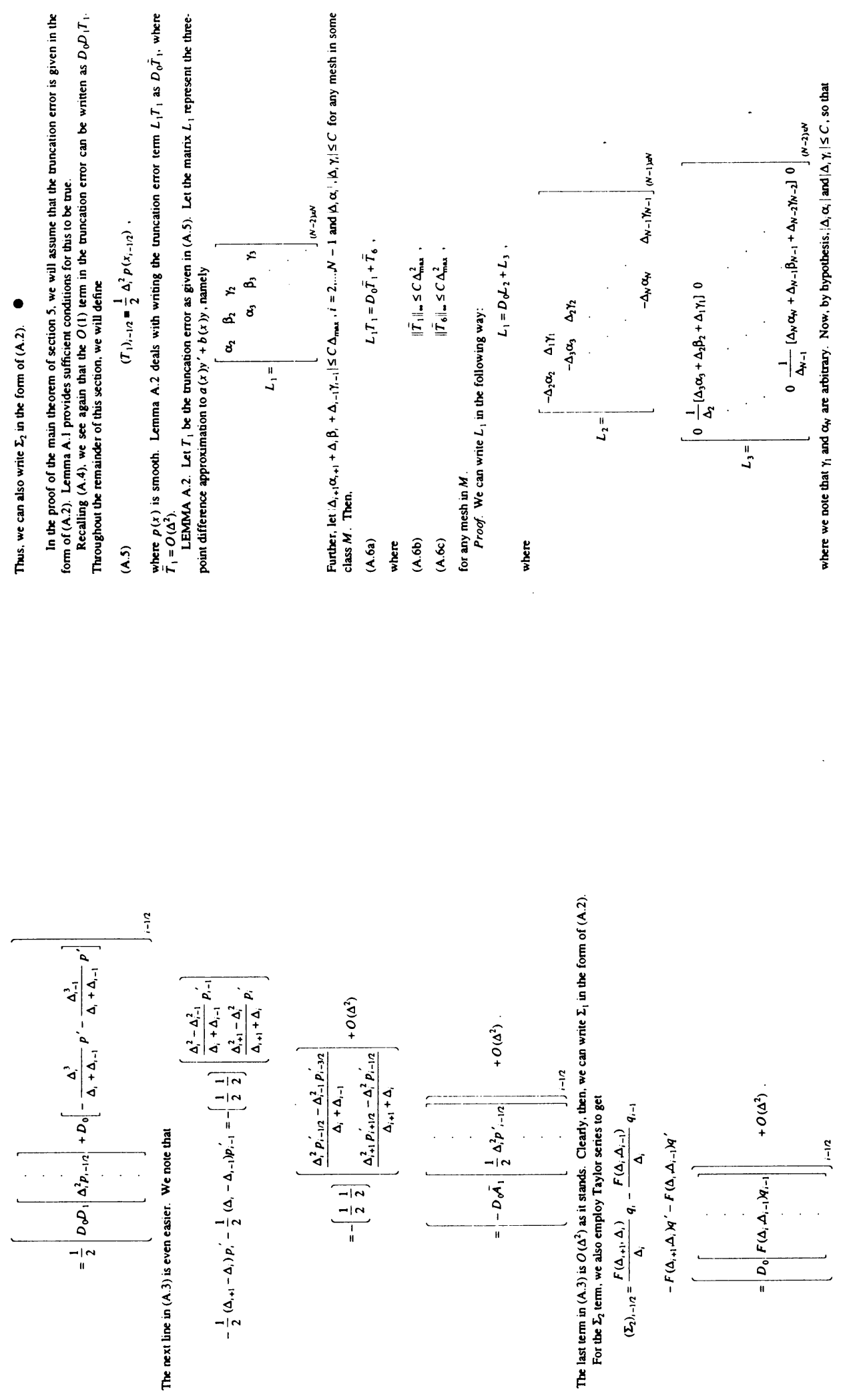

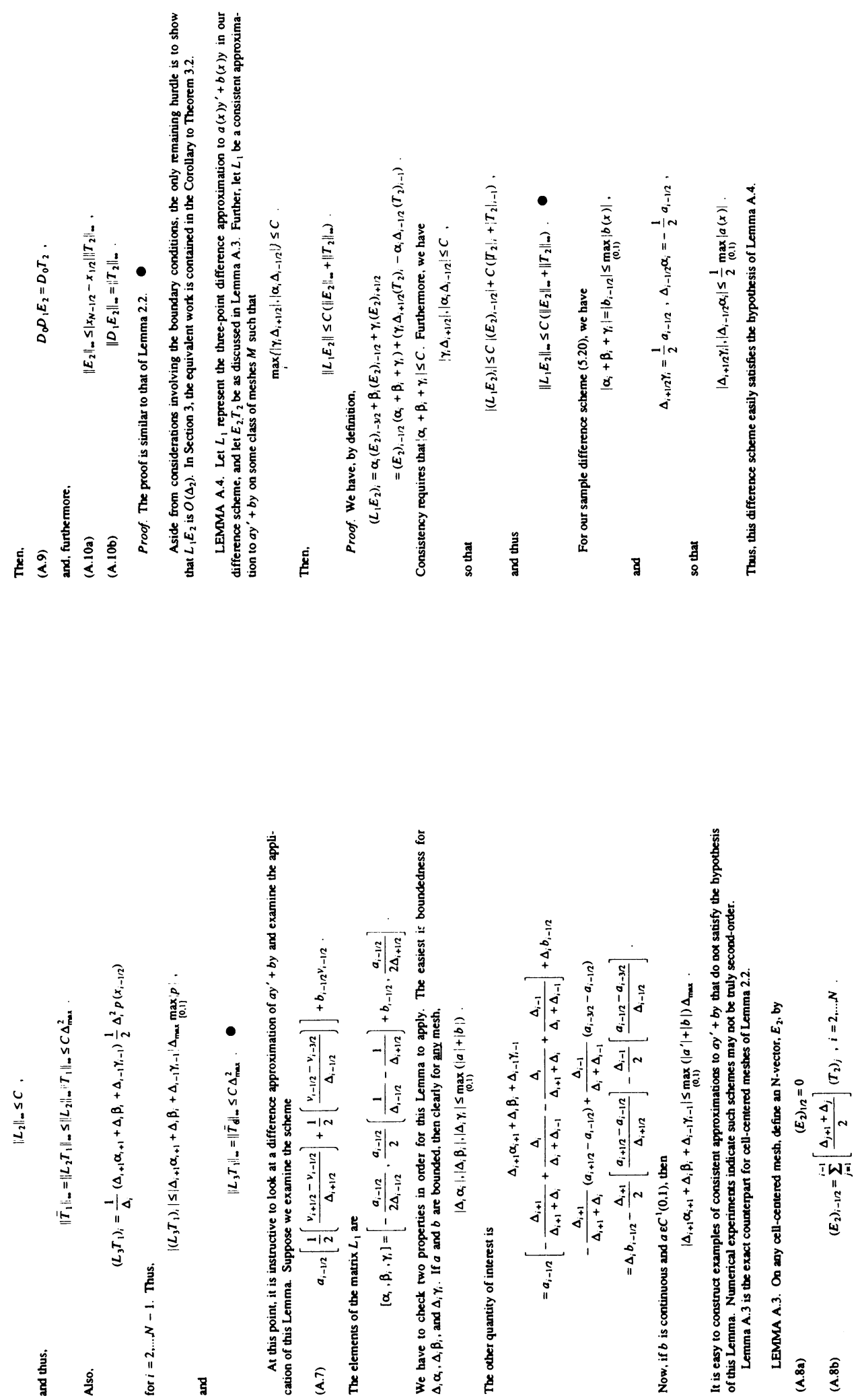